\title{
In silico integration of thousands of epigenetic datasets into 707 cell type regulatory annotations improves the trans-ethnic portability of polygenic risk scores
}

Tiffany Amariuta*1-5, Kazuyoshi Ishigaki*1-3,6 ${ }^{1-}$ Hiroki Sugishita ${ }^{7}$, Tazro Ohta ${ }^{8,9}$, Koichi Soumya Raychaudhuri ${ }^{1-5,18}$

${ }^{1}$ Center for Data Sciences, Harvard Medical School, Boston, Massachusetts, 02115, USA.

${ }^{2}$ Divisions of Genetics and Rheumatology, Department of Medicine, Brigham and Women's Hospital, Harvard Medical School, Boston, Massachusetts, 02115, USA.

${ }^{3}$ Program in Medical and Population Genetics, Broad Institute of MIT and Harvard, Cambridge, Massachusetts, 12 02142, USA.

$13{ }^{4}$ Department of Biomedical Informatics, Harvard Medical School, Boston, Massachusetts, 02115, USA.

$14{ }^{5}$ Graduate School of Arts and Sciences, Harvard University, Cambridge, Massachusetts, 02138, USA.

${ }^{6}$ Laboratory for Statistical and Translational Genetics, RIKEN Center for Integrative Medical Sciences, Kanagawa, 230-0045 Japan.

${ }^{7}$ Laboratory for Developmental Genetics, RIKEN Center for Integrative Medical Sciences (IMS), Kanagawa, Japan

${ }^{8}$ Medical Sciences Innovation Hub Program, RIKEN, Kanagawa, Japan.

${ }^{9}$ Database Center for Life Science, Joint Support-Center for Data Science Research, Research Organization of Information and Systems, Shizuoka, Japan.

${ }^{10}$ Laboratory of Genome Technology, Human Genome Center, Institute of Medical Science, The University of Tokyo, Tokyo, 108-8639 Japan.

${ }^{11}$ Laboratory of Clinical Genome Sequencing, Department of Computational Biology and Medical Sciences, Graduate School of Frontier Sciences, The University of Tokyo, Tokyo, 108-8639 Japan.

${ }^{12}$ Division of Molecular Pathology, Institute of Medical Science, The University of Tokyo, Tokyo, 108-8639 Japan

${ }^{13}$ Department of Epidemiology, Harvard T.H. Chan School of Public Health, Boston, MA 02115, USA.

${ }^{14}$ Department of Biostatistics, Harvard T.H. Chan School of Public Health, Boston, MA 02115, USA.

${ }^{15}$ Artificial Intelligence Medicine, Graduate School of Medicine, Chiba University, Chiba, Japan.

${ }^{16}$ Clinical Research Center, Shizuoka General Hospital, Shizuoka, 420-8527 Japan.

${ }^{17}$ The Department of Applied Genetics, The School of Pharmaceutical Sciences, University of Shizuoka, Shizuoka, 422-8526 Japan.

${ }^{18}$ Centre for Genetics and Genomics Versus Arthritis, Centre for Musculoskeletal Research, Manchester Academic Health Science Centre, The University of Manchester, Manchester, UK. 


\section{Abstract}

Poor trans-ethnic portability of polygenic risk score (PRS) models is a critical issue that may be partially due to limited knowledge of causal variants shared among populations. Hence,

52 leveraging noncoding regulatory annotations that capture genetic variation across populations

53 has the potential to enhance the trans-ethnic portability of PRS. To this end, we constructed a unique resource of 707 cell-type-specific IMPACT regulatory annotations by aggregating 5,345

55 public epigenetic datasets to predict binding patterns of 142 cell-type-regulating transcription

56 factors across 245 cell types. With this resource, we partitioned the common SNP heritability of

57 diverse polygenic traits and diseases from 111 GWAS summary statistics of European (EUR, average $\mathrm{N}=180 \mathrm{~K}$ ) and East Asian (EAS, average $\mathrm{N}=157 \mathrm{~K}$ ) origin. For 95 traits, we were able to identify a single IMPACT annotation most strongly enriched for trait heritability. Across traits, these annotations captured an average of $43.3 \%$ of heritability (se $=13.8 \%$ ) with the top $5 \%$ of SNPs. Strikingly, we observed highly concordant polygenic trait regulation between populations: the same regulatory annotations captured statistically indistinguishable SNP

63 heritability (fitted slope $=0.98$, se $=0.04$ ). Since IMPACT annotations capture both large and consistent proportions of heritability across populations, prioritizing variants in IMPACT regulatory

65 elements may improve the trans-ethnic portability of PRS. Indeed, we observed that EUR PRS models more accurately predicted 21 tested phenotypes of EAS individuals when variants were

67 prioritized by key IMPACT tracks (49.9\% mean relative increase in $R^{2}$ ). Notably, the

68 improvement afforded by IMPACT was greater in the trans-ethnic EUR-to-EAS PRS application

69 than in the EAS-to-EAS application (47.3\% vs $20.9 \%, P<1.7 \mathrm{e}-4)$. Overall, our study identifies a 
71 genetic data, and this has important implications for future risk prediction models that work

72 across populations.

\section{Introduction}

An important challenge for complex trait genetics is that there is no clear framework to transfer population-specific genetic data, such as GWAS results, to individuals of other ancestries ${ }^{1-3}$. The

78 importance of this challenge is accentuated by the fact that $80 \%$ of all genetic studies have

79 been performed using individuals of European ancestry, accounting for a minority of the

80 world's population ${ }^{4}$. This is exacerbated by the fact that population-specific linkage

81 disequilibrium (LD) between variants confounds inferences about causal cell types and variants

82 (Figure $1 \mathrm{~A})^{5-7}$. GWAS have the potential to revolutionize the clinical application and utility of

83 genetic data to the individual, exemplified by current polygenic risk score (PRS) models ${ }^{5,8-16}$.

84 However, while the utility of PRS models relies on accurate estimation of allelic effect sizes

85 from GWAS and benefits from genetic similarity between the test cohort and the GWAS cohort,

86 recent studies have explicitly observed a lack of trans-ethnic portability ${ }^{2,3,5,8,17,18}$. Previous

87 studies have extensively shown that functional annotations can improve PRS models when

88 learned and applied to the same population ${ }^{19,20}$, by introducing biologically-relevant priors on

89 causal effect sizes and compensating for inflation of association statistics by LD. However, the

90 potential for functional annotations to improve trans-ethnic PRS frameworks, where the

91 influences of population-specific LD are more profound, has not yet been extensively

92 investigated. 
However, designing functional annotations that may improve PRS models is challenging.

94 While the genetic variation of a complex trait likely regulates diverse biological mechanisms

95 genome-wide, such functional annotations must strike a balance of specificity, comprehensively

96 but precisely capturing large regulatory programs. Pinpointing these mechanisms is especially

97 difficult as genome-wide association studies (GWAS) have identified thousands of genetic

98 associations with complex phenotypes ${ }^{8,21-23}$. It has been estimated that about $90 \%$ of these

99 associations reside in protein noncoding regions of the genome, making their mechanisms

100 difficult to interpret ${ }^{24,25}$. Defining the etiology of complex traits and diseases requires

101 knowledge of phenotyping-driving cell types in which these associated variants act.

102 Transcription factors (TFs) are poised to orchestrate large polygenic regulatory programs as

103 genetic variation in their target regions can modulate gene expression, often in cell-type-

104 specific contexts ${ }^{26,27}$. Genomic annotations marking the precise location of TF-mediated cell

105 type regulation can be exploited to elucidate the genetic basis of polygenic traits. However,

106 currently there is no comprehensive catalogue of the binding profiles of the approximately

107 1,600 human TFs in every known cell type ${ }^{28}$. Moreover, existing TF ChIP-seq datasets are

108 limited to factors with effective antibodies and suffer from inter-experimental variation, noise,

109 and genomic bias ${ }^{29,30}$.

110 To overcome these challenges, we previously developed IMPACT, a genome-wide cell-

111 type-specific regulatory annotation strategy that models the epigenetic pattern around active

112 TF binding using linear combinations of functional annotations ${ }^{31}$. In rheumatoid arthritis (RA),

113 IMPACT CD4+ T cell annotations captured substantially more heritability than functional

114 annotations derived from single experiments, including TF and histone modification ChIP-seq ${ }^{6}$.

115 In this study, we expanded this approach by aggregating 5,345 functional annotations with 
116 IMPACT to create a powerful and generalizable resource of 707 cell-type-specific gene

117 regulatory annotations (Web Resources) based on binding profiles of 142 TFs across 245 cell

118 types (Figure 1B,C). This study builds on our previous study introducing IMPACT, in which we

119 created only 13 annotations (13 TFs) based on 515 functional annotations. Assuming that causal

120 variants are largely shared between populations ${ }^{2,21}$, we hypothesized that restricting PRS

121 models to variants within trait-relevant IMPACT annotations, which are more likely to have

122 regulatory roles and less likely to be confounded by LD, will especially improve their trans-

123 ethnic portability.

124 In this study, we identify key IMPACT regulatory annotations that capture genome-wide

125 polygenic mechanisms underlying a diverse set of complex traits, supported by enrichments of

126 genetic heritability, multi-ethnic marginal effect size correlation (a mechanism of improved

127 PRS), and improved trans-ethnic portability of PRS models (Figure 1D). Here, we defined and

128 employed our compendium of 707 IMPACT regulatory annotations to study polygenic traits and

129 diseases from 111 GWAS summary datasets of European (EUR) and East Asian (EAS) origin.

130 Assuming shared causal variants between populations, annotations that prioritize shared

131 regulatory variants must (1) capture disproportionately large amounts of genetic heritability in

132 both populations, (2) be enriched for multi-ethnic marginal effect size correlation, and (3)

133 improve the trans-ethnic applicability of population-specific PRS models. Using our

134 compendium of regulatory annotations, we identified key annotations for each polygenic trait

135 and demonstrated their utility in each of these three applications toward prioritization of

136 shared regulatory variants. Overall, this work improves the interpretation and trans-ethnic

137 portability of genetic data and provides implications for future clinical implementations of risk 138 prediction models. 
Figure 1

A

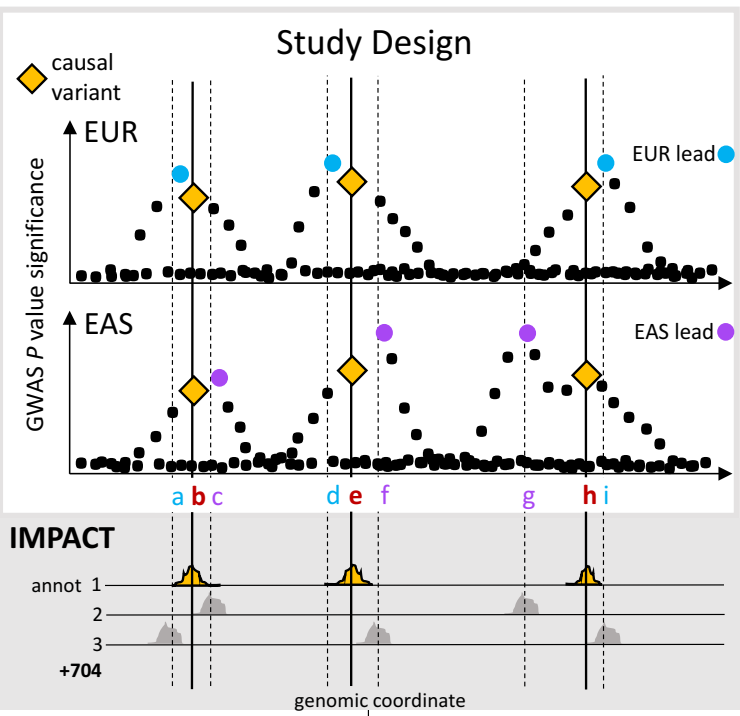

B

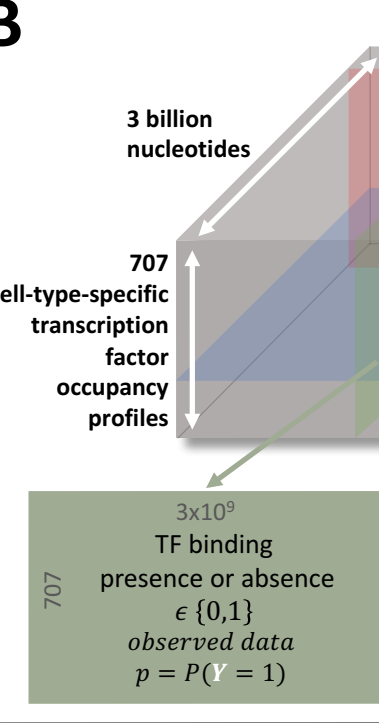

$\downarrow$

II. High trans-ethnic marginal effect size correlation

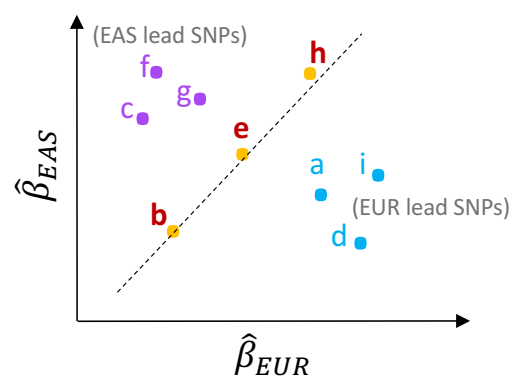

$$
\hat{\beta}_{E U R}
$$

5,345 epigenetic features

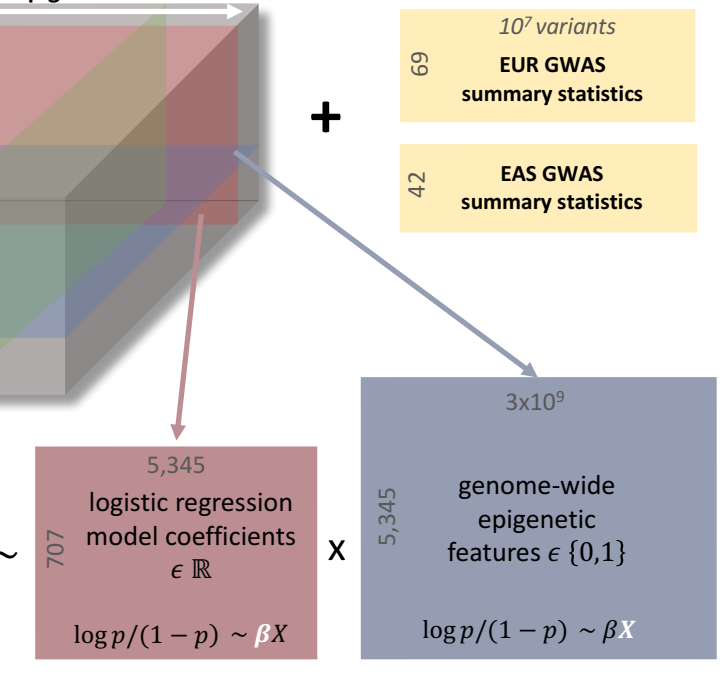

.
I. Heritability in shared regulatory elements

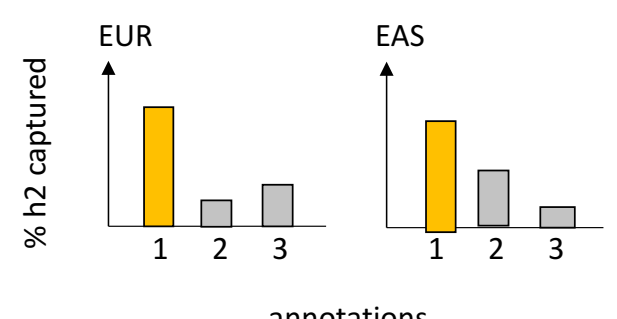

annotations
III. Trans-ethnic polygenic risk score models IMPACT PRS: EUR PRS model with $b, e, h$ standard PRS: EUR PRS model with a, d, i$$
\text { phenotype }
$$

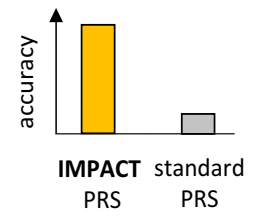

Figure 1 legend. Study design to identify regulatory annotations that prioritize regulatory variants in a multi-ethnic setting. A) Population-specific LD confounding and subsequent inflation of GWAS associations complicate the interpretation of summary statistics and transferability to other populations; functional data may help improve trans-ethnic genetic portability. B) Prism of functional data in IMPACT model: 707 genome-wide TF occupancy profiles (green), 5,345 genome-wide epigenomic feature profiles (blue), and fitted weights for these features (pink) to predict TF binding by logistic regression. Using IMPACT annotations, we investigate 111 GWAS summary datasets (yellow) of EUR and EAS origin. C) Compendium of 707 genome-wide cell-type-specific IMPACT regulatory annotations. D) Annotations that prioritize common regulatory variants must I) capture large proportions of heritability in both populations, II) account for consistent effect size estimations between populations and III) improve the transethnic application of PRS. 


\section{Results}

\section{Building a compendium of in silico gene regulatory annotations}

149 active TF binding evidenced by ChIP-seq, differentiating bound from unbound TF sequence

150 motifs using logistic regression. We derive this signature from 5,345 epigenetic and sequence

151 features, predominantly generated by ENCODE ${ }^{33}$ and Roadmap ${ }^{34}$ (Online Methods, ST2); these

152 data were drawn from diverse cell types, representing the biological range of the 707 candidate

153 models. IMPACT then probabilistically annotates the genome, e.g. on a scale from 0 to 1 ,

154 without using the TF motif, identifying regulatory regions that are similar to those that the TF

155 binds.

157 predict binding of the modeled TF, (2) share cell-type-specific characteristics with other tracks

158 of the same cell type, and (3) score cell-type-specifically expressed genes higher than

159 nonspecific genes. The 707 models that we defined had a high TF binding prediction accuracy

160 with mean AUPRC $=0.74(\mathrm{se}=0.008, \mathrm{SF} 2)$ using cross-validation. Annotations segregated by cell

161 type rather than by TF, excluding CTCF, suggesting a single TF may bind to different enhancers 
162 in different cell types (Figure 2A). Annotations of the same cell types were more strongly

163 correlated genome-wide (Pearson $r=0.56$, se $=0.06$ ) than annotations of different cell types

164 (Pearson $r=0.48$, se $=0.003$, difference of means $P<0.03$, SF2). Furthermore, the covariance

165 structure between TF ChIP-seq training datasets is similar to that of corresponding IMPACT

166 annotations, indicating that the IMPACT model does not introduce spurious correlations among

167 unrelated ChIP-seq datasets (SF2). Lastly, for nine different cell types, we examined cell-type-

168 specifically expressed genes from Finucane et $\mathrm{al}^{35}$ and corresponding differential expression $t$ -

169 statistics. We observed significantly larger IMPACT probabilities at SNPs in and near these genes

$170($ mean $=0.062$, se $=0.011)$ compared to genes that were generally expressed $($ mean $=0.045$, se

$171=0.006$; difference of means $P=0.024$, Figure 2B, SF2, Online Methods), suggesting that

172 IMPACT annotates relevant cell type regulatory elements.

Partitioning common SNP heritability of 111 GWAS summary statistics in EUR and EAS

177 for diverse polygenic traits and diseases. Throughout the text, we use five randomly selected

178 traits to exemplify our results: asthma, RA, prostate cancer (PrCa), mean corpuscular volume

179 (MCV), and body height. These included 69 from EUR participants ${ }^{31,36}$ (average $\mathrm{N}=180 \mathrm{~K}$,

180 average heritability $z$-score $=12.9,41 / 69$ from UK BioBank $)^{6,37}$ and 42 from EAS participants of

181 BioBank Japan ${ }^{3,38-40}$ (average $\mathrm{N}=157 \mathrm{~K}$, average heritability z-score $\left.=6.6\right)^{22}(\mathrm{ST} 3$ ). All of the

182 summary statistics used were generated from studies that had a sample size greater than

18310,000 individuals and also had a significantly non-zero heritability (z-score > 1.97). There are

18429 phenotypes for which we obtained summary statistics in both EUR and EAS. Although 10/29 


\section{Figure 2}

A Cell type distribution of 707 IMPACT annotations

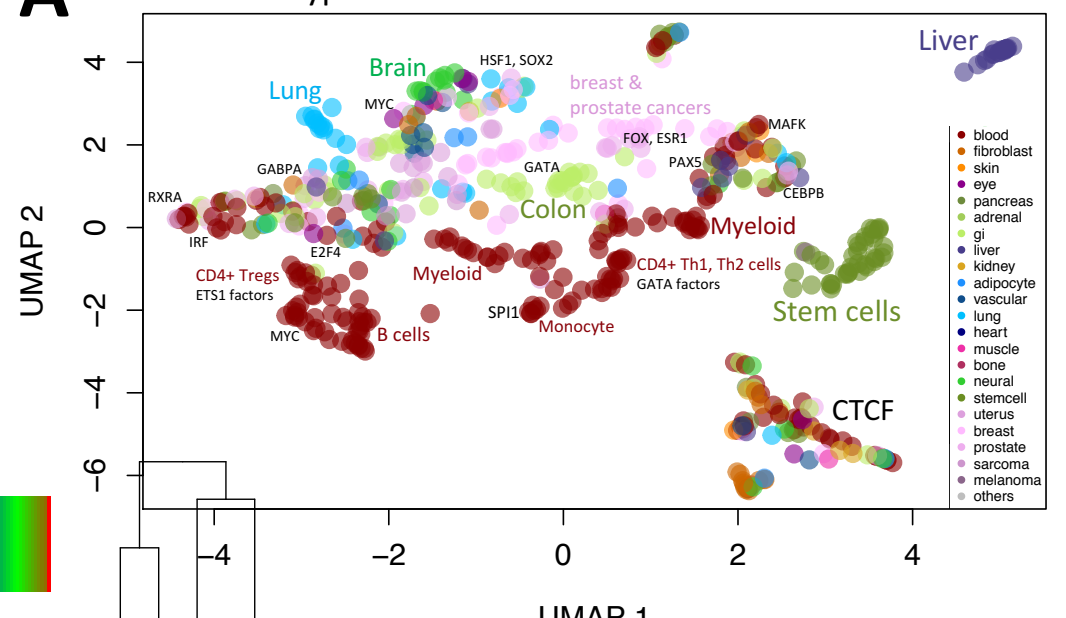

UMAP 1
B

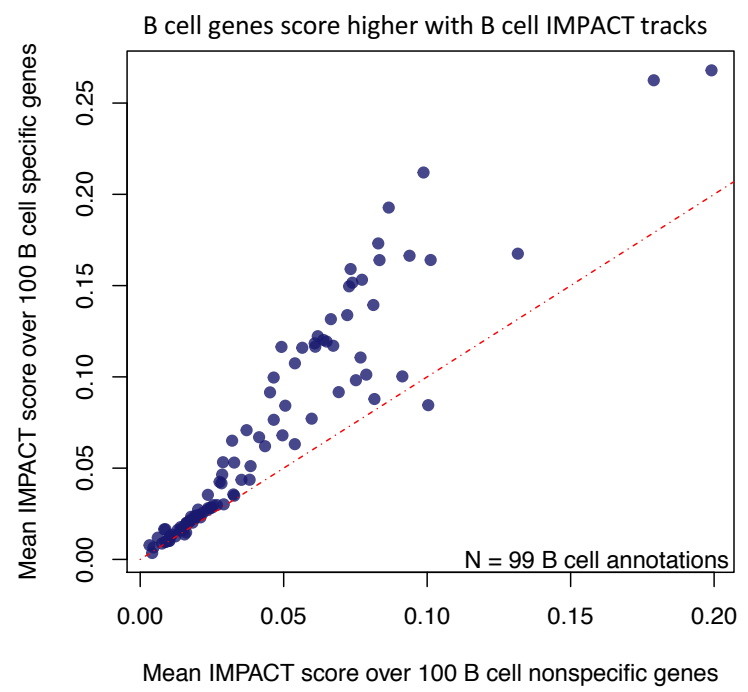

Colygenic trait associations across

D \begin{tabular}{l|l}
20 selected IMPACT annotations \\
\hline
\end{tabular}

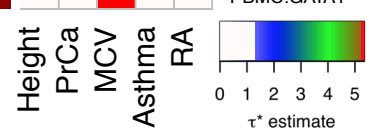
Heritability in the top $5 \%$ of SNPS

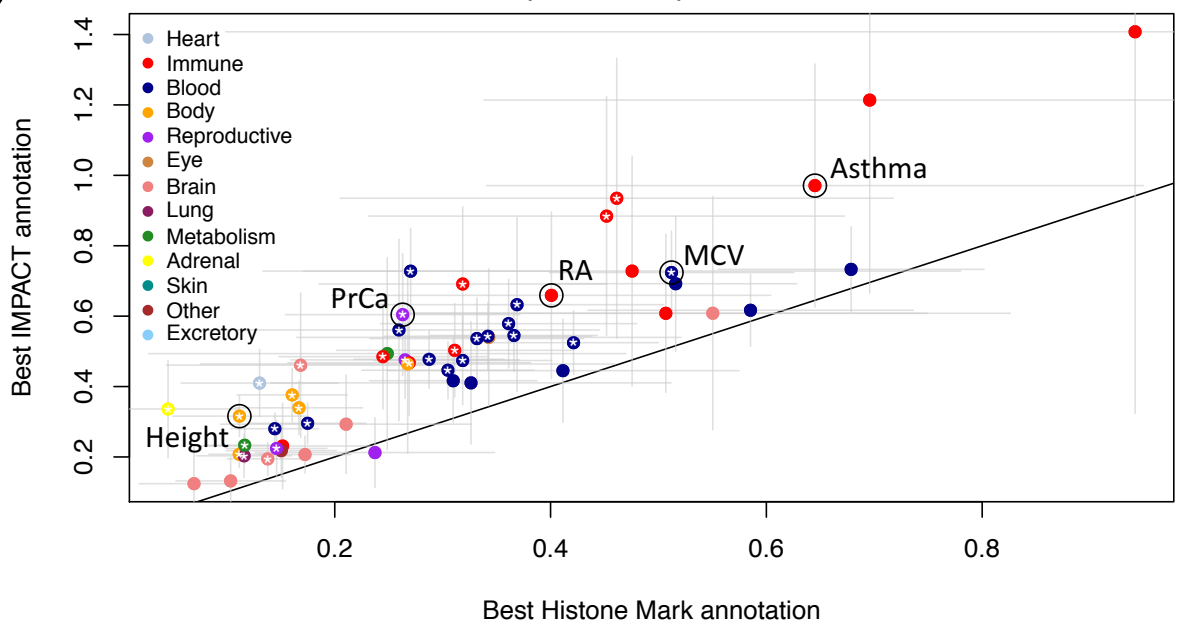

Figure 2 legend. IMPACT annotates relevant cell type regulatory elements. A) Low-dimensional embedding and clustering of 707 IMPACT annotations using uniform manifold approximation projection (UMAP). Annotations colored by cell type category; TF groups indicated where applicable. B) IMPACT annotates cell type specifically expressed genes with higher scores than nonspecific genes. C) Biologically distinct regulatory modules revealed by cell type-trait associations with significantly nonzero $\tau^{*}$ across 20 of 707 IMPACT regulatory annotations and 5 representative EUR complex traits, color indicates $-\log _{10}$ FDR $5 \%$ adjusted $P$ value of $\tau^{*}$. D) Lead IMPACT annotations capture more heritability than lead cell-type-specific histone modifications across 60 of 69 EUR summary statistics for which a lead IMPACT annotation was identified. ${ }^{*}$ indicates heritability estimate difference of means $P<0.05$. 
185 traits have a multi-ethnic genetic correlation $\left(R_{g}\right)$ significantly less than $1(P<0.05 / 29$ tested

186 traits), overall we observed high $R_{g}$ for most traits, supporting our assumption that causal

187 variants are generally shared across populations (Online Methods, SF3) ${ }^{41}$. At two extremes,

188 basophil count has a low multi-ethnic $R_{g}$ of $0.32(\mathrm{sd}=0.10)$, while atrial fibrillation has a high

189 multi-ethnic $R_{g}$ of 0.98 ( $\left.\mathrm{sd}=0.11\right)$, consistent with previous observations made using Popcorn,

190 but using different parameter estimation strategies (Online Methods) ${ }^{3}$.

191 We then partitioned the common SNP (minor allele frequency (MAF) $>5 \%$ ) heritability

192 of these 111 datasets using S-LDSC ${ }^{6}$ with an adapted baseline-LD model excluding cell-type-

193 specific annotations ${ }^{31,36}$ (SF3, Online Methods). Next, we tested each of the traits against each

194 of the 707 IMPACT annotations, assessing the significance of a non-zero $\tau^{*}$, which is defined as

195 the proportionate change in per-SNP heritability associated with a one standard deviation

196 increase in the value of the annotation (Online Methods) ${ }^{36}$. We observed that 95 phenotypes

197 had at least one significant annotation-trait association $\left(\tau^{*}>0\right.$, two-tailed FDR $<5 \%$, Ext. Data

198 1, Online Methods, ST4-8). Here, we highlight associations with EUR summary statistics for the

199 five exemplary phenotypes mentioned above: asthma, RA, PrCa, MCV, and height (Figure 2C).

200 Consistent with known biology, B and T cells were strongly associated with asthma ${ }^{42}, \mathrm{RA}^{43}$, and

$201 \mathrm{MCV}^{44,45}$ while other blood cell regulatory annotations predominantly derived from GATA

202 factors were also associated with MCV. Prostate cancer cell lines were associated with PrCa,

203 while many cell types including myoblasts ${ }^{46}$, fibroblasts ${ }^{47}$, and adipocytes ${ }^{48,49}$, lung cells, and

204 endothelial cells were associated with height, perhaps related to musculo-skeletal

205 developmental pathways.

206 For each trait, we defined the lead IMPACT regulatory annotation as the annotation

207 capturing the greatest per-SNP heritability, e.g. the largest while significant $\tau^{*}$ estimate (ST9). 
208 With their top 5\% of SNPs, lead IMPACT annotations captured an average of $43.3 \%$ heritability

209 (se $=13.8 \%$ ) across these 95 polygenic traits (SF4, Online Methods), with more than $25 \%$ of

210 heritability captured for two-thirds of the tested summary statistics (73/111 traits) and more

211 than $50 \%$ captured for $28 \%$ (31/111). Returning to our five exemplary phenotypes, with the top

$2125 \%$ of EUR SNPs, IMPACT captured $97.1 \%(\mathrm{sd}=17.6 \%)$ of asthma heritability with the T-bet Th1

213 annotation, $65.9 \%(\mathrm{sd}=12.1 \%)$ of RA heritability with the B cell TBP annotation, $60.4 \%(\mathrm{sd}=$

214 8.9\%) of PrCa heritability with the prostate cancer cell line (LNCAP) TFAP4 annotation, 72.4\%

$215(s d=6.0 \%)$ of MCV heritability with the GATA1 PBMC annotation, and lastly $31.6 \%(s d=3.0 \%)$

216 of height heritability with the lung MXI1 annotation (Figure 2D). While the observed association

217 between lung and height is not intuitive, within the MXI1 gene lies a genome-wide significant

218 variant associated with height ${ }^{50}$. Moreover, we captured significantly more heritability across

219 EUR traits using our expanded set of 707 IMPACT annotations (mean $=49.5 \%$, se $=12.0 \%$ )

220 compared to the 13 annotations in our previous study (mean $=32.3 \%$, se $=1.3 \%$, difference of

221 means $P=0.02$.

To demonstrate the value of IMPACT tracks, we compared them to annotations derived

223 from single experimental assays. For example, since each of the IMPACT tracks was trained on

224 TF ChIP-seq data, we directly compared the heritability captured by both data types. We

225 observed that the heritability captured by lead IMPACT annotations (mean $\tau^{*}=3.53$, se $=0.91$ )

226 was significantly greater than by the analogous TF ChIP-seq used in training (mean $\tau^{*}=1.71$, se

$227=0.94$, difference of means $P=0.02$ ). We also compared IMPACT tracks to histone marks, which

228 are commonly used to quantify cell type heritability ${ }^{6}$. From 220 publicly available cell-type-

229 specific histone mark ChIP-seq annotations of EUR SNPs ${ }^{6}$, we selected the lead histone mark

230 track for each of 69 EUR summary statistics. Restricting to the top 5\% of SNPs, we observed that 
231 the mean EUR heritability captured by lead IMPACT annotations $(49.5 \%$, se $=12.0 \%)$ was

232 significantly greater than by lead histone mark annotations $(28.4 \%$, se $=9.0 \%$, difference of

233 means $P=0.02$ ) (Figure 2D, ST10). For example, the lead IMPACT annotation for asthma

234 captured $1.5 \times$ more heritability than the best histone mark annotation (H3K27ac in CD4+ Th2),

235 capturing $64.2 \%$ ( $s d=15.5 \%$ ) of heritability. Similarly, IMPACT captured $1.7 x$ more RA

236 heritability than H3K4me3 in CD4+ Th17s; IMPACT captured 1.4x more MCV heritability than

237 H3K4me3 in CD34+ cells; IMPACT captured 2.3x more PrCa heritability than H3K4me3 in CD34+

238 cells; and IMPACT captured 3.1x more height heritability than H3K4me3 in lung cells. In terms

239 of $\tau^{*}$, IMPACT also captured more per-SNP heritability than histone marks: mean $\tau^{*}$ fold

$240 \quad$ change $=1.38 \times($ SF5).

241 Since some of our IMPACT annotations are similar to each other (SF2), we performed

242 serial conditional analyses in order to identify IMPACT annotations explaining heritability

243 independently from one another (Online Methods). This strategy might identify complex traits

244 for which several distinct biological mechanisms are independently regulated by genetic

245 variation. Indeed, we identified 30 EUR phenotypes and 8 EAS phenotypes with multiple

246 independent IMPACT associations (SF6, ST11-12). For example, four annotations were

247 independently associated with EUR PrCa: prostate (TFAP4), prostate (RUNX2), mesendoderm

248 (PDX1), and cervix (NFYB). Moreover, for seven EUR traits, three IMPACT annotations were

249 independently associated: height (adipocytes, fibroblasts, lung), neutrophil count (monocytes,

250 adipocytes, B cells), osteoporosis (myoblasts, mesenchymal stem cells, cervix), IBD (T cells and

251 two B cell annotations), platelet count (PBMCs, hematopoietic progenitors, muscle), systolic

252 blood pressure (endothelial, mesenchymal stem cells, fibroblasts), and white blood cell count (B

253 cells, adipocytes, hematopoietic progenitors). We found that the heritability z-score, an index 
254 correlated with the power of S-LDSC ${ }^{6}$, is strongly predictive of the number of independent

255 regulatory associations (linear regression $P<5.4 \mathrm{e}-9$ ), while sample size is not (linear regression

$256 P=0.19)(S F 7)$. Our findings suggest that multiple independent regulatory programs can

257 contribute to the heritability of complex traits, and we can detect them when phenotypes are

258 sufficiently heritable and the GWAS provide accurate effect size estimation.

260 Concordance of polygenic regulation between European and East Asian populations

Previous studies have shown concordance of polygenic effects between EUR and EAS individuals in $\mathrm{RA}^{1}$ and between EUR and African American individuals in $\mathrm{PrCa}^{51}$. However, to our

264 knowledge, the extent of these shared effects has not yet been comprehensively investigated

265 across many functional annotations and in diverse traits. Here, we quantified the SNP

266 heritability $\left(\tau^{*}\right)$ of 29 traits in EUR and EAS captured by a set of approximately 100 independent

267 IMPACT regulatory annotations (Figure 3B, SF8, Online Methods). Assuming shared causal

268 variants in EUR and EAS, IMPACT annotations that best prioritize shared genomic regions

269 regulating a phenotype presumably also disproportionately capture similar amounts of

270 heritability in both EUR and EAS (Figure 1D-I, Figure 3A). Briefly, we selected independent

271 annotations using an iterative pruning approach: for each trait, we ranked all annotations by $\tau^{*}$

272 and removed any annotation correlated with Pearson $r>0.5$ to the lead annotation and then

273 repeated. As IMPACT annotations are independent of population-specific factors including LD

274 and allele frequencies (SF3), they are poised to capture the genome-wide distribution of

275 regulatory variation in a population-independent manner. We observed that $\tau^{*}$ estimates

276 across annotations for EUR and EAS are strikingly similar, with a regression coefficient that is 
Figure 3
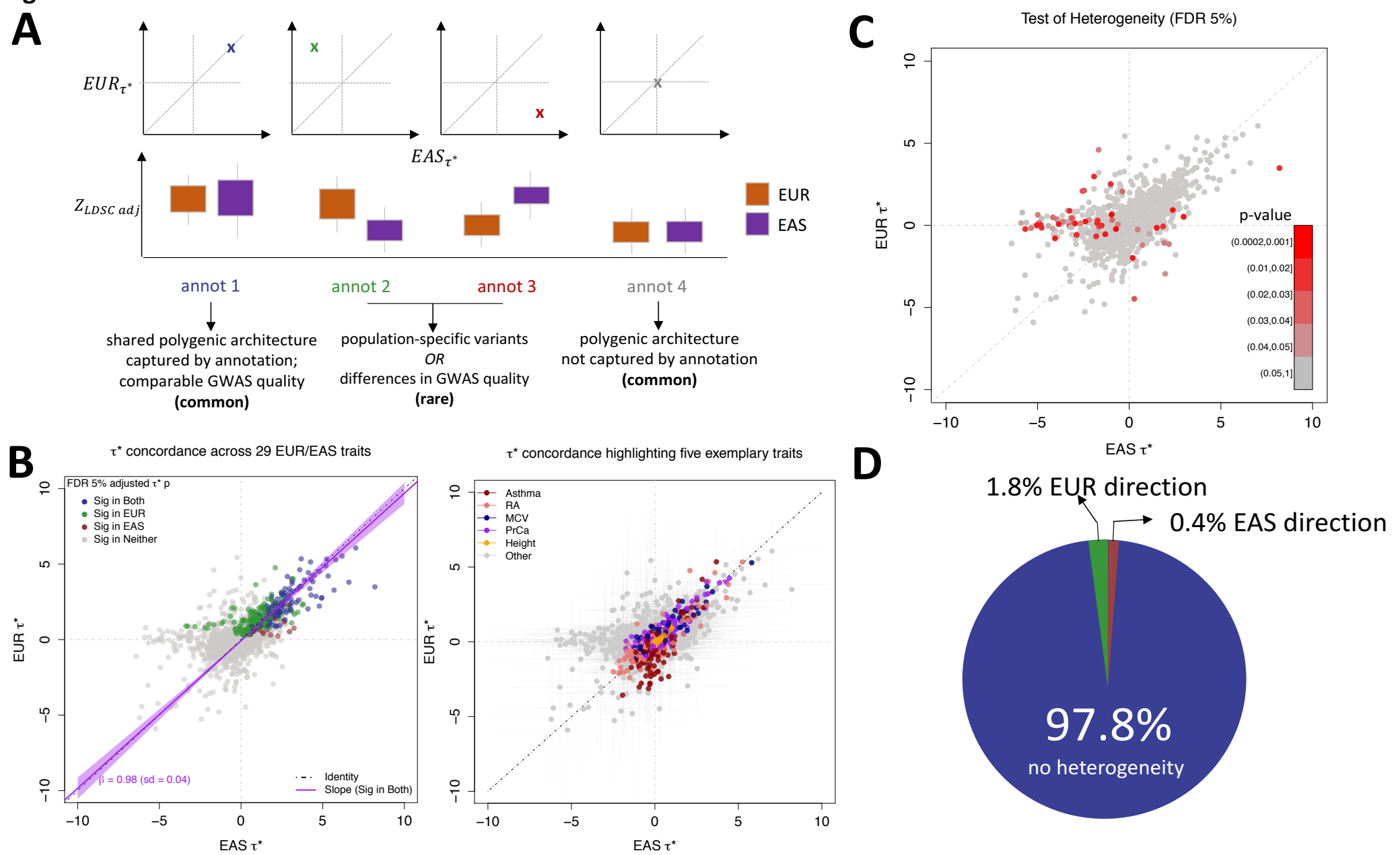

Figure 3 legend. Multi-ethnic concordance of regulatory elements defined by IMPACT. A) Illustrative concept of concordance versus discordance of $\tau^{*}$ between populations. Concordance implies a similar distribution of causal variants and effects captured by the same annotation. The implications of discordant $\tau^{*}$ are not as straightforward. B) Common per-SNP heritability ( $\tau^{*}$ ) estimate for sets of independent IMPACT annotations across 29 traits shared between EUR and EAS. Left: color indicates $\tau^{*}$ significance ( $\tau^{*}$ greater than 0 at 5\% FDR) in both populations (blue), significant in only EUR (green), significant in only EAS (red), significant in neither (gray). Line of best fit through annotations significant in both populations (dark purple line, $95 \% \mathrm{Cl}$ in light purple). Black dotted line is the identity line, $y=x$. Right: color indicates association to one of five exemplary traits. C) Heterogeneity test at 5\% FDR for annotationtrait associations between EUR and EAS. Color indicates significance of difference of means $P$ value. D) Heterogeneity test reveals $2.2 \%$ of all annotation-trait associations with significantly discordant $\tau^{*}$ estimates between populations. 
277 consistent with identity (slope $=0.98$, se $=0.04$ ). For example, we observed a strong Pearson

278 correlation of $\tau^{*}$ between EUR and EAS for asthma $(r=0.98), \mathrm{RA}(r=0.87), \mathrm{MCV}(r=0.96), \operatorname{PrCa}$

$279(r=0.90)$, and height $(r=0.96)$. Furthermore, we found that $97.8 \%$ of our $\tau^{*}$ estimates have no

280 evidence of population heterogeneity (FDR $P>0.05$ ) (Figure 3C). Among our five representative

281 traits, we observed only one instance of heterogeneity, in which the B cell SRF IMPACT

282 annotation captured RA heritability significantly more in EUR than in EAS (EUR $\tau^{*}=1.20$ (se =

$2830.40)$, EAS $\tau^{*}=-1.06(\mathrm{se}=0.46)$, difference of means $\left.P<2.0 \mathrm{e}-4\right)$. Overall, our results suggest

284 that regulatory variants in EUR and EAS populations are equally enriched within the same

285 classes of regulatory elements. This does not exclude the possibility of population-specific

286 variants or causal effect sizes, as evidenced by 10 traits with multi-ethnic genetic correlation

287 significantly less than 1. Rather, these results suggest that causal biology, including disease-

288 driving cell types and their regulatory elements, underlying polygenic traits and diseases, is

289 largely shared between these populations.

Models incorporating IMPACT functional annotations improve the trans-ethnic portability of

polygenic risk scores

PRS models have great clinical potential: previous studies have shown that individuals

295 with higher PRS have increased risk for disease ${ }^{8-12}$. In the future, polygenic risk assessment may

296 become as common as screening for known mutations of monogenic disease, especially as it

297 has been shown that individuals with severely high PRS may be at similar risk to disease as are

298 carriers of rare monogenic mutations ${ }^{12}$. However, since PRS heavily rely on GWAS with large

299 sample sizes to accurately estimate effect sizes, there is specific demand for the transferability 
300 of PRS from populations with larger GWAS to populations underrepresented by

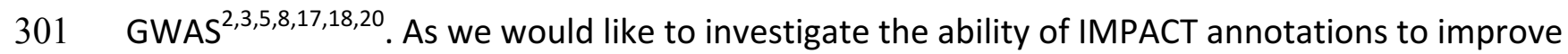

302 the trans-ethnic application of PRS, we chose pruning and thresholding (P+T) as our model ${ }^{3,8}$.

303 We elected to use P+T rather than LDpred ${ }^{5,20}$ or AnnoPred $^{19}$, which compute a posterior effect

304 size estimate for all SNPs genome-wide based on membership to functional categories. With

$305 \mathrm{P}+\mathrm{T}$, we can partition the genome by IMPACT-prioritized and deprioritized SNPs, whereas the

306 assumptions of the LDpred and AnnoPred models do not support the removal of variants,

307 making it difficult to directly assess improvement due to IMPACT prioritization. Moreover,

308 these models have not been explicitly designed or tested for the trans-ethnic application of PRS

309 and thus are beyond the scope of our work. We conventionally define PRS as the product of

310 marginal SNP effect size estimates and imputed allelic dosage (ranging from 0 to 2 ), summed

311 over M SNPs in the model. Conventional P+T utilizes marginal effect size estimates and

312 therefore is susceptible to selecting a tagging variant over the causal one guided by GWAS $P$

313 values that are inflated by LD. Therefore, we hypothesized that any observed improvement due

314 to incorporation of IMPACT annotations could result from prioritization of variants with higher

315 marginal multi-ethnic effect size correlation (Figure 1D-II).

316 Hence, we tested this hypothesis before assessing PRS performance. We selected 21 of

31729 summary statistics shared between EUR and EAS with an identified lead IMPACT association

318 in both populations. Then, using EUR lead IMPACT annotations for each trait (ST9), we

319 partitioned the genome three ways: (1) the SNPs within the top 5\% of the IMPACT annotation,

320 (2) the SNPs within the bottom 95\% of the IMPACT annotation, and (3) the set of all SNPs

321 genome-wide (with no IMPACT prioritization). We then performed stringent LD pruning $\left(r^{2}<0.1\right.$

322 from EUR individuals of phase 3 of 1000 Genomes $^{52}$ ), guided by the EUR GWAS $P$ value, to 
323 acquire sets of independent SNPs in order to compute a EUR-EAS marginal effect size estimate

324 correlation (Online Methods).

325 For example, in height, EUR-EAS effect size estimates of SNPs in the top 5\% partition

326 (Pearson $r=0.434$, Figure 4A) are 11.4-fold more similar than those in the bottom 95\%

327 partition $(r=0.038$, Figure 4B) and 3.31-fold more similar than the set of all SNPs $(r=0.131)$.

328 Meta-analyzed across the 21 traits, the marginal multi-ethnic effect size correlation among the

329 top 5\% of IMPACT SNPs was significantly greater than the set of all SNPs genome-wide, across

330 the 10 most lenient of 17 GWAS locus $P$ value thresholds examined (all difference of means $P<$

331 0.026) (Figure 4C-D). Furthermore, this observation was consistent across individual traits (SF9).

332 For comparison, we performed the same analysis using alternative annotations: lead

333 annotations from 513 cell-type-specifically expressed gene sets (SEG) ${ }^{35}$ and 220 cell-type-

334 specific histone mark annotations $(\mathrm{CTS})^{6}$ (SF10). Marginal effect size correlation with IMPACT

335 was comparable to CTS when comparing the top 5\% of SNPs to the set of all SNPs (difference of

336 means $P$ value $>0.05$ at 14 of $17 P$ value thresholds, SF11). Compared to SEG, IMPACT-selected

337 SNPs had a significantly greater correlation at 7 of $17 P$ value thresholds (all difference of means

$338 P$ value $<0.02$, SF11). Overall, our results suggest that we might anticipate improved trans-

339 ethnic portability of PRS models by prioritizing SNPs in key IMPACT annotations.

341 Finally, we addressed our hypothesis that IMPACT annotations improve the trans-ethnic

342 portability of PRS (Figure 1D-III). For each of the 21 previously analyzed traits, we built a PRS

343 using effect size estimates from EUR summary statistics and applied it to predict phenotypes of

344 EAS individuals from BioBank Japan (BBJ) (Figure 5A). Here, we compare two PRS models, both

345 blind to any EAS genetic or functional information and removing SNPs with LD $r^{2}>0.2$, 


\section{Figure 4}

A

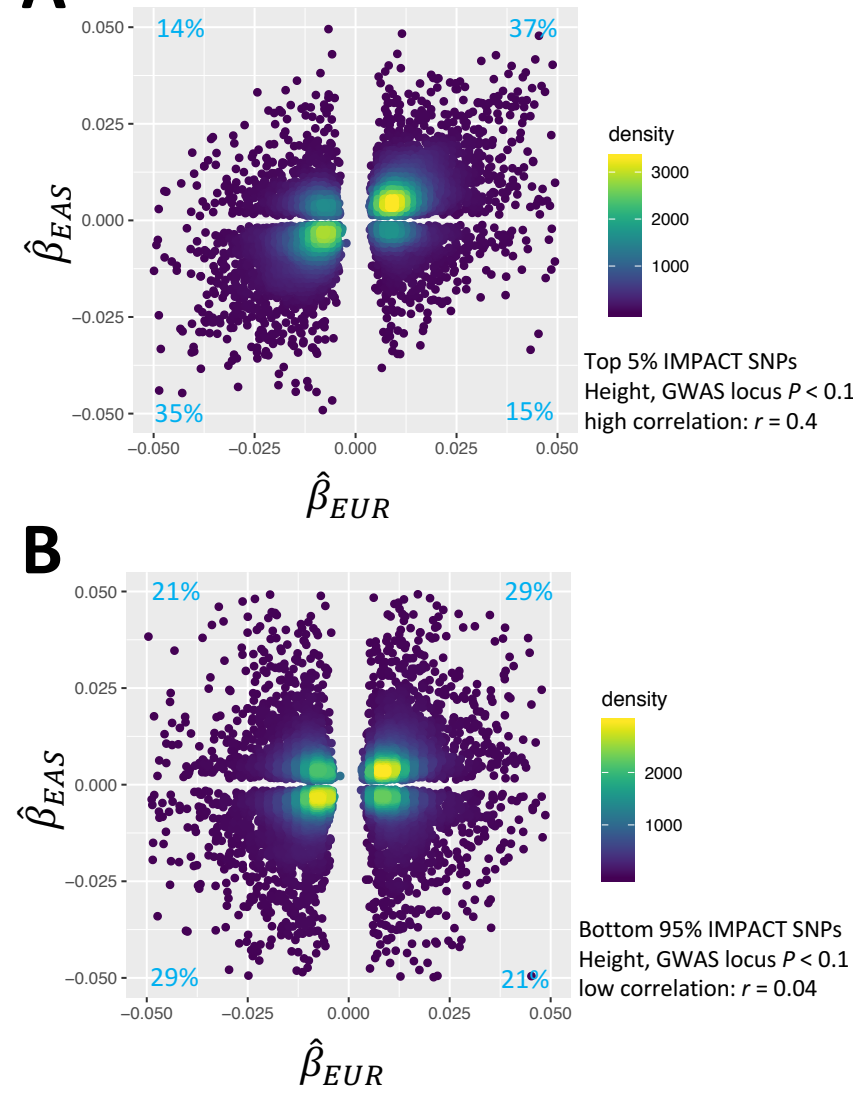

C

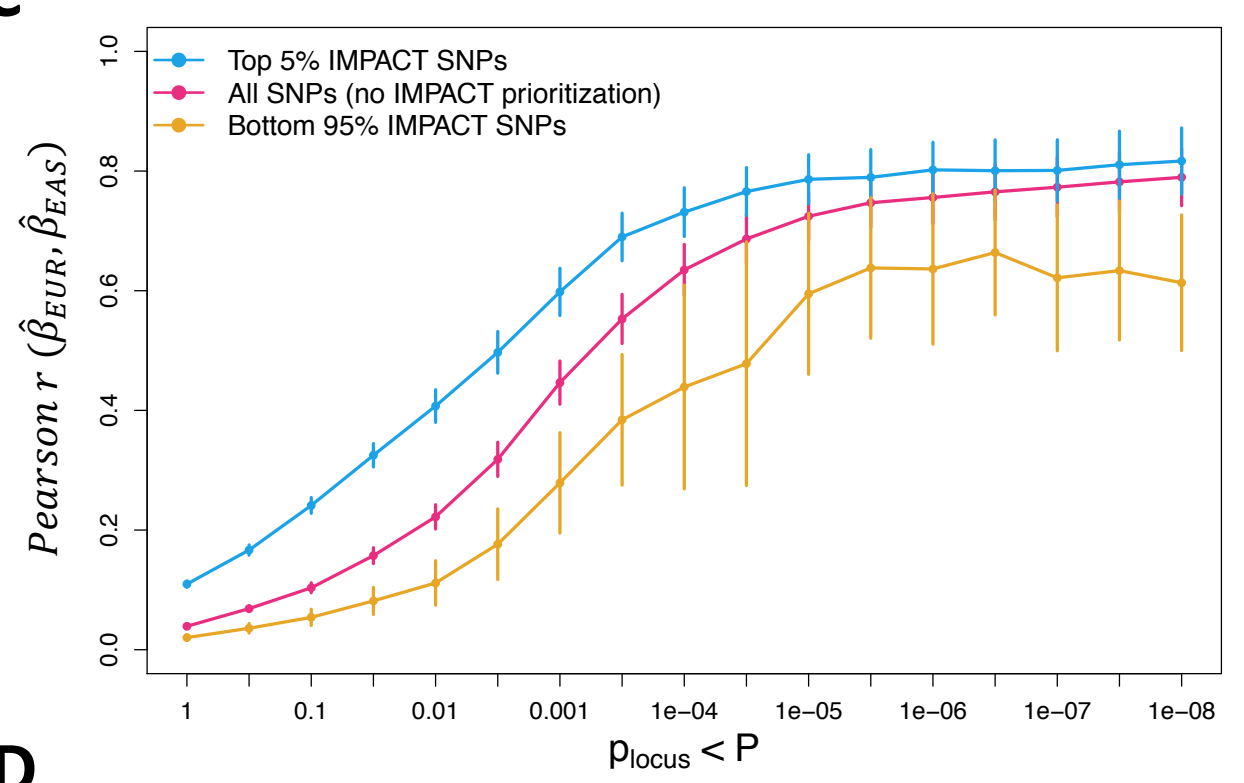

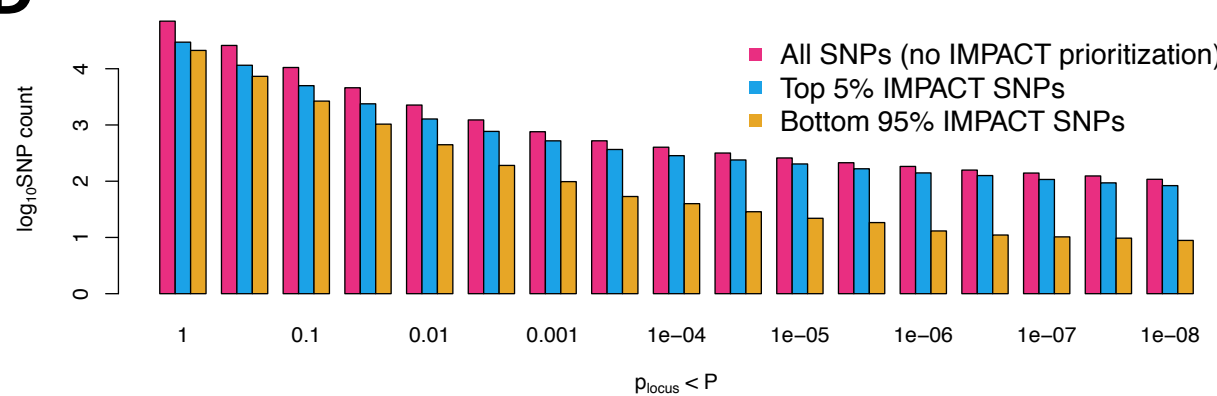

Figure 4 legend. Mechanism by which IMPACT prioritization of shared regulatory variants might improve trans-ethnic PRS performance. A) Estimated effect sizes of variants from genome-wide EUR and EAS height summary statistics in the top $5 \%$ of the lead IMPACT annotation for EUR height. Proportions of variants in each quadrant indicated in light blue. B) Estimated effect sizes from genome-wide EUR and EAS height summary statistics of variants in the bottom $95 \%$ of the same lead IMPACT annotation for height; mutually exclusive with SNPs in A). C) Meta-analysis of multi-ethnic marginal effect size correlations between populations across 21 traits shared between EUR and EAS cohorts over 17 GWAS $P$ value thresholds (with reference to the EUR GWAS). D) Number of SNPs ( $\log _{10}$ scale) at each $P$ value threshold for each partition of the genome corresponding to $C$ ). 

according to European individuals from phase 3 of 1000 Genomes $^{52}$ : (i) standard P+T PRS and (ii) functionally-informed P+T PRS using a subset of SNPs prioritized by the lead EUR IMPACT annotation (Online Methods). In functionally-informed PRS models, for each trait separately, we a priori selected the subset of top-ranked IMPACT SNPs (top 1\%, 5\%, 10\%, or 50\%) which explained the closest to $50 \%$ of total trait heritability (Online Methods). For all PRS models, we report results from the most accurate model across nine EUR GWAS $P$ value thresholds. phenotypic variance than standard PRS (49.9\% mean relative increase in $R^{2}$, Figure 5B, SF12,

ST13-15). The mean phenotypic variance explained across traits by functionally-informed PRS

$355\left(R^{2}=2.1 \%\right.$, se $\left.=0.2 \%\right)$ was greater than by standard PRS $\left(R^{2}=1.5 \%\right.$, se $\left.=0.1 \%\right)$. For 20 of 21

356 traits, e.g. excluding basophil count, IMPACT-informed PRS significantly outperformed standard

357 PRS (difference of means $P<0.01$ ). Using 10,000 bootstraps of the PRS sample cohort, we

358 found that the IMPACT-informed PRS $R^{2}$ estimate was consistently greater than the standard

359 PRS estimate for the same 20 traits (all bootstrap $P<0.004$, ST15). We observed the largest

360 improvement for RA from $R^{2}=1.4 \%(\mathrm{sd}=0.33 \%)$ in the standard PRS versus $R^{2}=4.1 \%(\mathrm{sd}=$

$3610.53 \%$, difference of means $P<7.7 \mathrm{e}-10$ ) in the functionally-informed PRS using the B cell TBP

362 IMPACT annotation. For asthma, $R^{2}=0.37 \%(\mathrm{sd}=0.10 \%)$ in the standard PRS versus $R^{2}=0.75 \%$

$363(\mathrm{sd}=0.14 \%, P<8.5 \mathrm{e}-4)$ in the functionally-informed PRS. For $\mathrm{MCV}, R^{2}=3.0 \%(\mathrm{sd}=0.10 \%)$ in

364 the standard PRS versus $R^{2}=4.1 \%(\mathrm{sd}=0.12 \%, P<1.9 \mathrm{e}-25)$ in the functionally-informed PRS.

365 For $\operatorname{PrCa}, R^{2}=4.5 \%(\mathrm{sd}=0.36 \%)$ in the standard PRS versus $R^{2}=6.4 \%(\mathrm{sd}=0.45 \%, P<2.4 \mathrm{e}-6)$ in 366 the functionally-informed PRS. For height, $R^{2}=4.2 \%$ ( $\mathrm{sd}=0.10 \%$ ) in the standard PRS versus $R^{2}$ $367=5.6 \%(\mathrm{sd}=0.12 \%, P<1.2 \mathrm{e}-37)$ in the functionally-informed PRS. 
For our five representative traits asthma, RA, MCV, PrCa, and height, we further compared functionally-informed PRSEUR using IMPACT to models using cell-type-specifically expressed genes (SEG) and cell-type-specific histone modification tracks (CTS) ${ }^{6,35}$ (Figure 5C,

371 ST16). Across all of the five traits, models using IMPACT explained significantly greater

372 phenotypic variance (mean $R^{2}=4.2 \%$, se $=0.3 \%$ ) than SEG $(0.9 \%$, se $=0.1 \%$, all difference of

373 means $P<9.9 \mathrm{e}-11)$. While IMPACT generally outperformed CTS $\left(R^{2}=2.6 \%\right.$, se $=0.2 \%$, difference

374 of means meta $P<1.2 \mathrm{e}-8)$, this observation was only individually consistent with 3 of 5 traits

375 (difference of means $P<9.3 e-8$ ). We performed a similar bootstrap analysis as above, yielding

376 similar results; for only RA and asthma did IMPACT-PRS not produce consistently greater

$377 R^{2}$ estimates than CTS-PRS (ST16).

Functionally-informed PRS might to some extent compensate for population-specific LD differences between populations. Hence, we hypothesized that IMPACT-informed PRS would improve standard PRS moreso in the trans-ethnic prediction framework, in which EUR PRS

381 models predict EAS phenotypes, than in a within-population framework, in which EAS PRS

382 models predict EAS phenotypes. Here, we define within-population PRS as PRSEAS and trans-

383 ethnic PRS as PRSEUR to avoid confusion. In order to directly compare PRS model improvements

384 between PRSEAS and PRSEUR, we evaluated prediction accuracy on the same individuals. Briefly,

385 we partitioned the BBJ cohort to reserve 5,000 individuals for PRS testing, derived GWAS

386 summary statistics from the remaining individuals, and performed P+T PRS modeling and

387 prediction as done above (Figure 5D, SF13-15, ST17-18, Online Methods). For functionally-

388 informed PRSEAS, we selected lead IMPACT annotations from S-LDSC results using GWAS

389 summary statistics, as done above, on the partition of the BBJ cohort excluding the 5,000 PRS

390 test individuals. We defined improvement as the percent increase in $R^{2}$ from standard to 
391 functionally-informed PRS; therefore, differences in PRS performance due to intrinsic factors,

392 such as GWAS power or genotyping platform, cancel out. In both scenarios, we observed

393 significant non-zero improvements: averaged across the 21 traits in the trans-ethnic setting

394 (mean percent increase in $R^{2}=47.3 \%$, se $=8.1 \%, P<2.7 e-9$ ) and in the within-population

395 setting (mean percent increase in $R^{2}=20.9 \%$, se $=6.6 \%, P<7.5 \mathrm{e}-4$ ). Indeed, this revealed a

396 significantly greater improvement in the trans-ethnic than in the within-population application

397 (difference of means $P<1.7 \mathrm{e}-4$, Figure 5E).

398 Overall, our results reveal that functional prioritization of SNPs using IMPACT

399 significantly improves both trans-ethnic and within-population PRS models, but is especially

400 advantageous for the trans-ethnic application of PRS. In conclusion, our results nominate the

401 prioritization of SNPs according to functional annotations, especially using IMPACT, as a

402 potential tentative solution for the lack of trans-ethnic portability of PRS models. While

403 individuals of European ancestry dominate current genetic studies, population-nonspecific cell-

404 type-specific IMPACT annotations can help transfer highly powered EUR genetic data to study

405 still underserved populations.

406

407 Discussion

408

In this study, we created a compendium of 707 cell-type-specific regulatory annotations

410 (Web Resources) capturing disproportionately large amounts of polygenic heritability in 95

411 complex traits and diseases in EUR and EAS populations. We then proposed a three-step

412 framework to assess how well prioritization of regulatory variants with functional data can

413 improve multi-ethnic genetic comparisons. First, we showed that heritability-enriched 


\section{Figure 5}

A

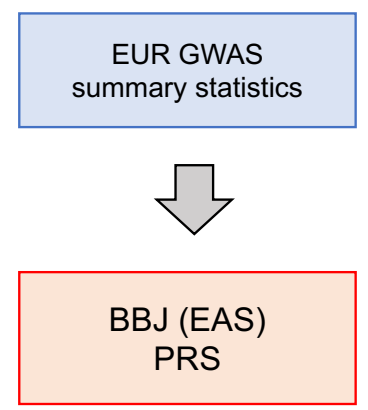

D

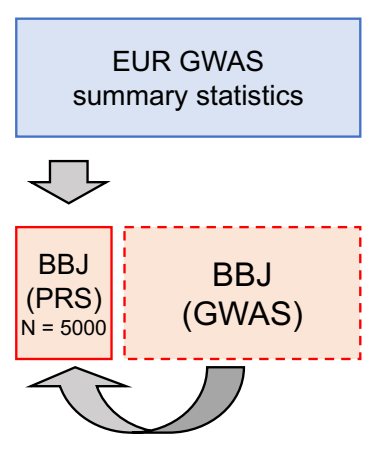

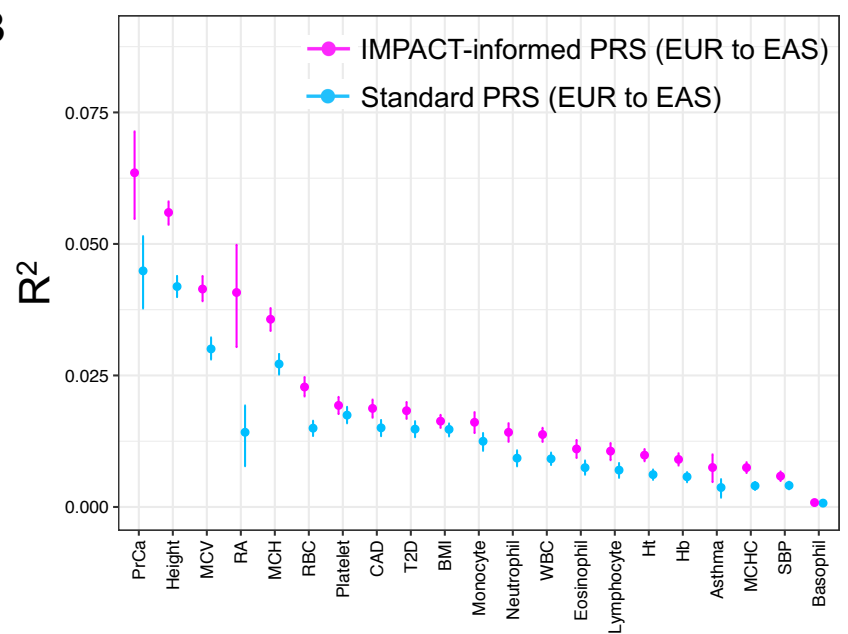

$\mathbf{E}$

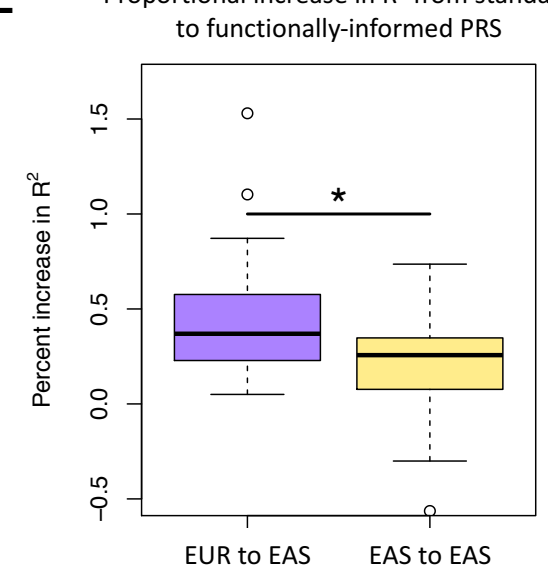

C

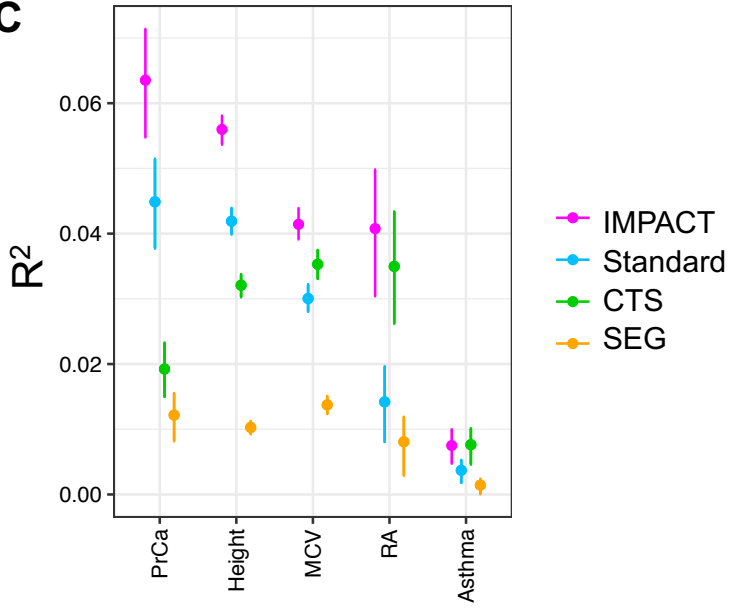

Figure 5 legend. Identifying shared regulatory variants with IMPACT annotations to improve the trans-ethnic portability of PRS. A) Study design applying EUR summary statistics-based PRS models to all individuals in the BBJ cohort. (B) Phenotypic variance $\left(R^{2}\right)$ of BBJ individuals explained by EUR PRS using two methods: functionally-informed PRS with IMPACT (pink) and standard PRS (blue). Error bars indicate $95 \% \mathrm{Cl}$ calculated via 1,000 bootstraps. C) Phenotypic variance $\left(\mathrm{R}^{2}\right)$ of $\mathrm{BBJ}$ individuals across 5 exemplary traits explained by EUR IMPACT annotations relative to lead cell-type-specific histone modification annotations (CTS) and lead cell-typespecifically expressed gene sets (SEG). Error bars indicate $95 \% \mathrm{Cl}$ calculated via 1,000 bootstraps. D) Study design to compare transethnic (EUR to EAS) to within-population (EAS to EAS) improvement afforded by functionally-informed PRS models. For each trait, 5,000 randomly selected individuals from BBJ designated as PRS samples. Remaining BBJ individuals used for GWAS to derive EAS summary statistics-based PRS; no shared individuals between GWAS samples and PRS samples. E) Improvement from standard PRS to functionally-informed PRS compared between trans-ethnic (EUR to EAS) and within-population models (EAS to EAS) using the study design in $\mathrm{D})$. 
414 regulatory elements between EUR and EAS populations capture indistinguishable proportions

415 of heritability across 29 complex traits. Second, we showed that functional prioritization of

416 variants selects those with more highly correlated marginal effect sizes between populations;

417 this might explain the improvement driven by functional prioritization in P+T PRS models which

418 use marginal effect sizes. Third, we showed that variant prioritization with IMPACT annotations

419 results in consistently improved PRS prediction accuracy, especially for the trans-ethnic

420 application; potentially due to overcoming large population-specific influences such as LD, an

421 important challenge of multi-population models.

422 Designing genetic models for each complex trait or disease that capture risk for the full

423 diversity of the human population will be challenging. This necessitates approaches that

424 effectively transfer predictive genetic information from well studied populations to less well

425 studied populations. Our work provides insight into the potential clinical implementation of PRS

426 and broader genetic applications that aim to integrate multi-ethnic data. We argue for the use

427 of biologically diverse IMPACT annotations to capture relevant genetic signal and compensate,

428 to some extent, for differences in LD across populations. While we did not assess a PRS model

429 using meta-analyzed summary statistics from two or more populations in this study, we believe

430 that this approach could be effective, especially for populations with limited GWAS sample size.

431 We believe that IMPACT may prioritize phenotype-driving regulatory variation. We have

432 shown IMPACT to be more effective at capturing genetic variation of complex traits than

433 commonly used functional annotations such as experimentally-derived cell-type-specific

434 histone marks or gene sets. We hypothesize the utility of IMPACT comes from 1) cell-type-

435 specificity of TF binding models which locate key classes of regulatory elements and 2) the

436 integration of thousands of experimentally-derived annotations, which presumably removes 
437 noise and enriches for biological signal present in each individual annotation. Here, we did not

438 demonstrate the potential utility of IMPACT to perform functional fine-mapping to reduce

439 credible sets beyond our previous work ${ }^{31}$, due to lack of sufficient gold standards with causal

440 experimental validation and the limitation to genome-wide significant variants. The specific

441 application of IMPACT in multi-ethnic fine-mapping needs to be further investigated.

442 We must consider several important limitations of our work. First, our functional

443 insights are limited to cell types with publicly available TF ChIP-seq data, lacking ones that are

444 rarer or more difficult to assay. In the future, the cell-type-specific functional training data for

445 IMPACT may be replaced by newer experimental strategies to map enhancers. For example,

446 high-throughput CRISPR screens paired with assays for open chromatin could be used to

447 precisely redefine the regulatory landscape. Second, we used multi-ethnic data to argue for the

448 utility of our approach. However, the robustness of multi-ethnic comparisons for a given

449 phenotype rely on properties surrounding the recruitment of individuals or the exact

450 genotyping platform used in various biobanks, which may result in cohort-bias that inflates

451 within-population PRS prediction accuracy. For example, BBJ is a disease ascertainment cohort,

452 in which each individual has any one of 47 common diseases ${ }^{53,54}$; therefore, BBJ control

453 samples are not comparable to healthy controls of UKBB. Other biases may arise from clinical

454 differences in phenotyping. Also, we only considered a single non-EUR population in this study,

455 while the disparity in trans-ethnic portability and hence resulting benefit from functional

456 annotations may be greater in other non-EUR populations.

457 In conclusion, we demonstrated that IMPACT annotations improve the comparison of

458 genetic data between populations and trans-ethnic portability of PRS models using ancestrally

459 unmatched data. While a long-term goal of the field must be to diversify GWAS and other 
460 genetic studies in non-European populations, it is imperative that genetic models be developed

461 that work in multiple populations. Such initiatives will necessitate the use of population-

462 independent functional annotations, such as IMPACT, in order to capture shared biological

463 mechanisms regulated by complex genetic variation.

464

465 Supplemental Data

466 See Supplement.pdf and Supplementary_Tables.xIsx

467

468 Online Methods

469 See Online_Methods.pdf

470

471 Acknowledgements

472 This work is supported in part by funding from the National Institutes of Health (NHGRI T32

473 HG002295, UH2AR067677, 1U01HG009088, U01 HG009379, and 1R01AR063759) and the Doris

474 Duke Charitable Foundation grant \#2013097.

475

476 Declaration of Interests

477 The authors declare no competing financial interests.

478

479 Web Resources

480

481 1. IMPACT 707 annotations:

482 https://github.com/immunogenomics/IMPACT/tree/master/IMPACT707

483 2. IMPACT Github repository: https://github.com/immunogenomics/IMPACT 
3. HOMER: http://homer.ucsd.edu/homer/motif/

4. S-LDSC: https://github.com/bulik/ldsc

5. 1000 Genomes: http://www.internationalgenome.org/

6. Cell-type-specifically expressed gene set annotations and LD scores: https://data.broadinstitute.org/alkesgroup/LDSCORE/LDSC SEG Idscores/

7. Cell-type-specific histone modification ChIP-seq datasets: https://data.broadinstitute.org/alkesgroup/LDSCORE/

References

1. Kichaev, G. \& Pasaniuc, B. Leveraging Functional-Annotation Data in Trans-ethnic FineMapping Studies. Am. J. Hum. Genet. 97, 260-271 (2015).

2. Lam, M. et al. Comparative genetic architectures of schizophrenia in East Asian and European populations. doi:10.1101/445874

3. Martin, A. R. et al. Clinical use of current polygenic risk scores may exacerbate health disparities. Nat. Genet. 51, 584-591 (2019).

4. Sirugo, G., Williams, S. M. \& Tishkoff, S. A. The Missing Diversity in Human Genetic Studies. Cell 177, 26-31 (2019).

5. Vilhjálmsson, B. J. et al. Modeling Linkage Disequilibrium Increases Accuracy of Polygenic Risk Scores. Am. J. Hum. Genet. 97, 576-592 (2015). association summary statistics. Nat. Genet. 47, 1228-1235 (2015). 
7. Bulik-Sullivan, B. K. et al. LD Score regression distinguishes confounding from polygenicity in genome-wide association studies. Nat. Genet. 47, 291-295 (2015).

8. International Schizophrenia Consortium et al. Common polygenic variation contributes to risk of schizophrenia and bipolar disorder. Nature 460, 748-752 (2009).

511 9. Chatterjee, N., Shi, J. \& García-Closas, M. Developing and evaluating polygenic risk prediction models for stratified disease prevention. Nat. Rev. Genet. 17, 392-406 (2016).

513 10. Stahl, E. A. et al. Bayesian inference analyses of the polygenic architecture of rheumatoid arthritis. Nat. Genet. 44, 483-489 (2012).

515 11. Chatterjee, N. et al. Projecting the performance of risk prediction based on polygenic analyses of genome-wide association studies. Nat. Genet. 45, 400-5, 405e1-3 (2013).

517 12. Khera, A. V. et al. Genome-wide polygenic scores for common diseases identify individuals with risk equivalent to monogenic mutations. Nat. Genet. 50, 1219-1224 (2018).

13. Schumacher, F. R. et al. Association analyses of more than 140,000 men identify 63 new prostate cancer susceptibility loci. Nat. Genet. 50, 928-936 (2018).

521 14. Sharp, S. A. et al. Development and Standardization of an Improved Type 1 Diabetes Genetic Risk Score for Use in Newborn Screening and Incident Diagnosis. Diabetes Care 42,

524 15. Kullo, I. J. et al. Incorporating a Genetic Risk Score Into Coronary Heart Disease Risk Estimates: Effect on Low-Density Lipoprotein Cholesterol Levels (the MI-GENES Clinical Trial). Circulation 133, 1181-1188 (2016). Atherosclerosis and Greater Relative Benefit From Statin Therapy in the Primary Prevention Setting. Circulation 135, 2091-2101 (2017). 
17. Márquez-Luna, C., Loh, P.-R., South Asian Type 2 Diabetes (SAT2D) Consortium, SIGMA Type 2 Diabetes Consortium \& Price, A. L. Multiethnic polygenic risk scores improve risk prediction in diverse populations. Genet. Epidemiol. 41, 811-823 (2017).

533 18. Duncan, L. et al. Analysis of polygenic risk score usage and performance in diverse human populations. Nat. Commun. 10, 3328 (2019).

19. Hu, Y. et al. Leveraging functional annotations in genetic risk prediction for human complex diseases. PLoS Comput. Biol. 13, e1005589 (2017).

20. Márquez-Luna, C. et al. Modeling functional enrichment improves polygenic prediction accuracy in UK Biobank and 23andMe data sets. bioRxiv 375337 (2018).

21. Okada, Y. et al. Genetics of rheumatoid arthritis contributes to biology and drug discovery. Nature 506, 376-381 (2014).

22. Kanai, M. et al. Genetic analysis of quantitative traits in the Japanese population links cell types to complex human diseases. Nature Genetics 50, 390-400 (2018).

544 23. Yengo, L. et al. Meta-analysis of genome-wide association studies for height and body mass index in $~ 700000$ individuals of European ancestry. Hum. Mol. Genet. 27, 3641-3649 (2018).

24. Schaub, M. A., Boyle, A. P., Kundaje, A., Batzoglou, S. \& Snyder, M. Linking disease associations with regulatory information in the human genome. Genome Res. 22, 17481759 (2012).

25. Maurano, M. T. et al. Systematic localization of common disease-associated variation in

552 26. Reshef, Y. A. et al. Detecting genome-wide directional effects of transcription factor 
553 binding on polygenic disease risk. Nat. Genet. 50, 1483-1493 (2018).

554 27. Liu, X., Li, Y. I. \& Pritchard, J. K. Trans Effects on Gene Expression Can Drive Omnigenic $555 \quad$ Inheritance. Cell 177, 1022-1034.e6 (2019).

556 28. Lambert, S. A. et al. The Human Transcription Factors. Cell 172, 650-665 (2018).

557 29. Teytelman, L., Thurtle, D. M., Rine, J. \& van Oudenaarden, A. Highly expressed loci are 558 vulnerable to misleading ChIP localization of multiple unrelated proteins. Proc. Natl. Acad.

$559 \quad$ Sci. U. S. A. 110, 18602-18607 (2013).

560 30. Skene, P. J. \& Henikoff, S. An efficient targeted nuclease strategy for high-resolution $561 \quad$ mapping of DNA binding sites. Elife 6, (2017).

562 31. Amariuta, T. et al. IMPACT: Genomic Annotation of Cell-State-Specific Regulatory Elements 563 Inferred from the Epigenome of Bound Transcription Factors. Am. J. Hum. Genet. 104, $564 \quad$ 879-895 (2019).

565 32. Kawakami, E., Nakaoka, S., Ohta, T. \& Kitano, H. Weighted enrichment method for 566 prediction of transcription regulators from transcriptome and global chromatin

33. ENCODE Project Consortium. An integrated encyclopedia of DNA elements in the human genome. Nature 489, 57-74 (2012).

34. Roadmap Epigenomics, C. et al. Heravi-428 Moussavi A, Kheradpour P, Zhang Z, Wang J, et

573 35. Finucane, H. K. et al. Heritability enrichment of specifically expressed genes identifies 574 disease-relevant tissues and cell types. Nat. Genet. 50, 621-629 (2018).

575 36. Gazal, S. et al. Linkage disequilibrium-dependent architecture of human complex traits 
shows action of negative selection. Nat. Genet. 49, 1421-1427 (2017).

37. Buniello, A. et al. The NHGRI-EBI GWAS Catalog of published genome-wide association studies, targeted arrays and summary statistics 2019. Nucleic Acids Res. 47, D1005-D1012 (2019).

38. Akiyama, M. et al. Characterizing rare and low-frequency height-associated variants in the Japanese population. Nat. Commun. 10, 4393 (2019).

39. Ishigaki, K., Akiyama, M., Kanai, M. \& Takahashi, A. Large scale genome-wide association study in a Japanese population identified 45 novel susceptibility loci for 22 diseases.

40. Akiyama, M. et al. Genome-wide association study identifies 112 new loci for body mass index in the Japanese population. Nat. Genet. 49, 1458-1467 (2017).

41. Brown, B. C., Ye, C. J., Price, A. L. \& Zaitlen, N. Transethnic Genetic-Correlation Estimates from Summary Statistics. Am. J. Hum. Genet. 99, 76-88 (2016).

42. Drake, L. Y. et al. B cells play key roles in th2-type airway immune responses in mice

43. Amariuta, T., Luo, Y., Knevel, R., Okada, Y. \& Raychaudhuri, S. Advances in genetics toward exposed to natural airborne allergens. PLoS One 10, e0121660 (2015). identifying pathogenic cell states of rheumatoid arthritis. Immunol. Rev. (2019). doi:10.1111/imr.12827

44. Buttari, B., Profumo, E. \& Riganò, R. Crosstalk between red blood cells and the immune system and its impact on atherosclerosis. Biomed Res. Int. 2015, 616834 (2015).

45. Anderson, H. L., Brodsky, I. E. \& Mangalmurti, N. S. The Evolving Erythrocyte: Red Blood Cells as Modulators of Innate Immunity. J. Immunol. 201, 1343-1351 (2018).

598 46. Lui, J. C. \& Baron, J. Mechanisms limiting body growth in mammals. Endocr. Rev. 32, 422- 
440 (2011).

600 47. Maier, A. B., van Heemst, D. \& Westendorp, R. G. J. Relation between body height and 601 replicative capacity of human fibroblasts in nonagenarians. J. Gerontol. A Biol. Sci. Med. 602 Sci. 63, 43-45 (2008).

603 48. Murphy, R. A. et al. Adipose tissue, muscle, and function: potential mediators of associations between body weight and mortality in older adults with type 2 diabetes. Diabetes Care 37, 3213-3219 (2014).

49. Heymsfield, S. B., Gallagher, D., Mayer, L., Beetsch, J. \& Pietrobelli, A. Scaling of human body composition to stature: new insights into body mass index. Am. J. Clin. Nutr. 86, 82-

50. Kichaev, G. et al. Leveraging Polygenic Functional Enrichment to Improve GWAS Power. Am. J. Hum. Genet. 104, 65-75 (2019).

51. Gusev, A. et al. Atlas of prostate cancer heritability in European and African-American men

612 pinpoints tissue-specific regulation. Nat. Commun. 7, 10979 (2016).

613 52. Gibbs, R. A. et al. A global reference for human genetic variation. Nature 526, 68-74 (2015).

615 53. Nagai, A. et al. Overview of the BioBank Japan Project: Study design and profile. J. $616 \quad$ Epidemiol. 27, S2-S8 (2017).

617 54. Hirata, M. et al. Cross-sectional analysis of BioBank Japan clinical data: A large cohort of 618 200,000 patients with 47 common diseases. J. Epidemiol. 27, S9-S21 (2017). 


\section{Online Methods}

\section{Data}

TF ChIP-seq data. We previously collected 3,181 publicly available transcription factor (TF) chromatin immunoprecipitation (ChIP) datasets derived from human primary cells or cell lines.

We downloaded raw sequencing data in SRA format from NCBI GEO, then converted the data to FASTQ format using the SRA Toolkit function fastq-dump, used FastQC for quality assessment of sequencing reads, and finally mapped reads to the human genome (hg19/GRCh37) with Bowtie2 [v2.2.5] using default parameters. All ChIP-seq datasets were matched to corresponding control data from which peaks were called with macs [v2.1] with q value $<0.01$ under a bimodal model, producing 3,181 bed file-formatted files ${ }^{1,2}$. The 1,542 datasets selected for use with our IMPACT model framework (see below) are listed with accession codes in ST1.

Genome-wide annotation data. We augmented our set of 515 publicly available epigenomic and sequence feature annotations from our previous study ${ }^{3}$ with 116 personally curated datasets from NCBI, 2,593 ENCODE histone ChIP-seq datasets and 2,121 ENCODE open chromatin DNase-seq datasets ${ }^{4}$, all publicly available at the accessions provided in ST2. All files were collected in 6-column standard bed file format. This augmentation brought the total number of features to 5,345 .

21 Genome-wide association data. We collected publicly available summary statistics data for 111 genome-wide association studies (GWAS) across separate cohorts of East Asian and European 
23 individuals ${ }^{5-7}$. East Asian GWAS data were collected from Biobank Japan (BBJ) while European

24 GWAS data were collected from either UKBioBank (UKBB) or the GWAS catalog, referred to as

25 PASS (publicly available summary statistics) (ST3). All GWAS summary statistics were

26 reformatted to be compatible with S-LDSC (see below) and thus contained the following

27 information for each SNP (per row): rsID, A1 (reference allele), A2 (alternative allele), GWAS

28 sample size (effective sample size per SNP, may vary with genotyping), chi-square statistic, z-

29 score. For multi-ethnic genetic correlation and polygenic risk score prediction, all GWAS

30 summary statistics were reformatted to contain the SNP ID (chr_position_A1_A2),

31 chromosome, base pair, A1, A2, effect size estimate, effect size estimate standard error, and $P$ -

32 value.

Cell-type-specifically expressed gene set (SEG) and cell-type-specific histone modification

35 (CTS) annotations. We downloaded 513 publicly available SEG annotations for European SNPS

36 from phase 3 of 1000 Genomes accompanied by pre-computed LD scores (see Web

37 Resources) ${ }^{8}$. SEG annotations are binary and thus each SNP is designated a 1 or a 0 , indicating

38 that the SNP does or does not lie, respectively, within $100 \mathrm{~kb}$ of the gene body of the

39 corresponding gene set ${ }^{8}$. We downloaded 220 publicly available CTS annotations of peak data

40 in bed file format, from which we annotated European SNPs from phase 3 of 1000 Genomes $^{9}$

41 and used S-LDSC to compute LD scores (see Web Resources) ${ }^{7}$. These annotations are also

42 binary, in which case each SNP is designated a 1 or a 0 , indicating that the SNP does or does not

43 like, respectively, within the peak of histone modification. 
45 BioBank Japan data. For PRS analysis, we utilized phenotype and genotype data of the BioBank 46 Japan Project $(\mathrm{BBJ})^{10,11}$. All of the calculations related to PRS were conducted on the RIKEN

47 computing server. BBJ is a biobank that collaboratively collects DNA and serum samples from

4812 medical institutions in Japan. This project recruited approximately 200,000 patients with the

49 diagnosis of at least one of 47 diseases. Informed consent was obtained from all participants by

50 following the protocols approved by their institutional ethical committees. We obtained

51 approval from the ethics committees of the RIKEN Center for Integrative Medical Sciences and

52 the Institute of Medical Sciences at the University of Tokyo.

\section{Statistical Methods}

IMPACT Model. We implemented our previously defined model to predict TF binding on a motif

57 site. This model regresses the likelihood $(p)$ of a binding event on the epigenomic profile of the

58 motif site, in a logistic regression framework over $j$ epigenomic features as follows:

$$
\log \left(\frac{p}{1-p}\right)=\beta_{0}+\beta_{1} X_{1}+\beta_{2} X_{2}+\ldots+\beta_{j} X_{j}
$$

60 We use a weighted average of ridge and lasso regularization terms in the objective function to

61 restrict the magnitude of fit coefficients and enforce sparsity to reduce overfitting, respectively,

62 as follows:

63

64

$$
\operatorname{argmin}_{\beta}=\left(\|Y-X \beta\|^{2}+\frac{1}{2}(1-\alpha)\|\beta\|^{2}+\alpha\|\beta\|\right)
$$


Training IMPACT. We trained an IMPACT model for each unique cell type-TF pair present in our

67 data collection. Our collection consists of 3,181 TF ChIP-seq profiles, representing 442 TFs, 296

68 cell types, and 24 tissues. The IMPACT model requires that the assayed TF has a distinct binding

69 motif and so we removed all ChIP-seq datasets corresponding to a TF that did not have a known

70 sequence motif in MEME, Jaspar, or Transfac databases. This resulted in 1,542 TF ChIP-seq

71 profiles across 142 TFs, 245 cell types, 23 tissues, and 728 unique combinations of TFs and cell

72 types. As we did in our previous study ${ }^{3}$, we merged experiments of the same TF-cell type

73 combination by taking the union of the peaks. We next identified motif sites bound by a TF by

74 using HOMER [v4.8.3 $]^{12}$ to scan ChIP-seq peaks for motif matches exceeding the empirically

75 determined motif detection threshold. Similarly, we identified motif sites not bound by a TF by

76 using HOMER to scan the entire genome for sequence matches. 21 of these models did not

77 contain sufficient overlap between TF sequence motifs and ChIP-seq peaks which would lead to

78 underfitting in the logistic regression (fewer than 7), thereby resulting in 707 total possible

79 IMPACT annotations. We then trained 707 IMPACT models using up to 1,000 TF-bound

80 sequence motifs (evidenced by ChIP-seq) and 10,000 unbound sequence motifs. For each of

81707 TF-cell type pairs, we learned a predictive model of TF binding and annotated SNPs

82 genome-wide for both EUR and EAS populations, with a mean regulatory probability per

83 nucleotide of 0.02 (se $=7.5 e-4)$.

84

85 Assessing cell type specificity of IMPACT tracks. We acquired lists of specifically expressed

86 genes in 9 different cell types: T cells, B cells, fibroblasts, monocytes, brain, liver, colon,

87 prostate, and breast according to differential gene expression $t$-statistics from previous work ${ }^{8}$, 
specifically labeled as T.4+8int.Th, B.Fo.LN, Cells_Transformed_fibroblasts, Mo.6C+II-.LN, Brain_Cortex, Liver, Colon_Transverse, Prostate, Breast_Mammary_Tissue, respectively from either ImmGen or GTEx databases. Large and positive $t$-statistics represent greater specificity of gene expression in the target cell type, large but negative $t$-statistics represent specifically repressed genes, and $t$-statistics near 0 represent nonspecific gene expression, representing commonly expressed genes. For each cell type, we selected the 100 genes with highest $t$ statistics, e.g. specifically expressed (SE) genes, and 100 genes such that $-0.5<t$-statistic $<0.5$, e.g. not specifically expressed genes (NS). For each cell type separately, we collected all related IMPACT annotations from the compendium of 707 total annotations. Then for each annotation separately, we computed the average IMPACT score over all EUR SNPs from phase 3 of 1000 Genomes within $2 \mathrm{~kb}$ of each SE or NS gene body. Finally, we computed the average across all 100 SE and 100 NS genes, separately.

Partitioning heritability with S-LDSC. We applied S-LDSC $[\mathrm{v} 1.0 .0]^{7}$ to partition the common (MAF > 5\%) SNP heritability of 111 polygenic traits and diseases, with significantly non-zero heritability estimates $(P<0.05)$. We partitioned heritability using a customized version of the baselineLD model, in which we excluded cell-type-specific regulatory annotations (as we would be testing the enrichment of such annotations from IMPACT). In total, we used 69 cell-typenonspecific baselineLD annotations and added one or more IMPACT annotations to the model to test for cell-type-specific enrichment. We use three metrics to evaluate how well our IMPACT annotations capture polygenic heritability: enrichment ${ }^{7}$, the proportion of heritability explained by the top $5 \%$ of SNPs ${ }^{7}$, and per-annotation standardized effect size, $\tau^{* 6}$. Briefly, 
enrichment is defined as the proportion of common SNP heritability divided by the genome-

111 wide proportion of SNPs in the annotation, for continuous annotations this is the average

112 annotation value across SNPs. $\tau$ * represents the average per-SNP heritability of a category of

113 SNPs, where a single SNP may claim membership to one or more categories. $\tau^{*}$ is defined as the

114 proportionate change in per-SNP heritability associated with a one standard deviation increase

115 in the value of the annotation. The sum of the $\tau^{*}$ over categories of SNPs equals the total

116 estimated heritability of the trait. $\tau^{*}$ has units of heritability and is comparable between traits,

117 annotations, and populations, because it is normalized for the total heritability (indicative of

118 the power of the GWAS), the dispersion of the annotation values (annotation size), and the

119 number of common SNPs (population-specific) considered in the model, respectively. $\tau$, the

120 precursor of $\tau^{*}$, is the coefficient estimated in the S-LDSC regression. $\tau$ and $\tau^{*}$ are conditionally

121 dependent on the provided baselineLD annotations. Therefore, the $\tau^{*}$ estimate for an IMPACT

122 annotation is considered a measure of cell-type-specific or annotation-specific SNP heritability,

123 as the remaining annotations in the model (baselineLD) are not cell-type-specific. Significance

124 of $\tau^{*}$ is computed using a $z$-test of how different the $\tau^{*}$ estimate is from 0 ; the significance of

125 strictly positive $\tau^{*}$ estimates are reported in our study.

127 Measuring heritability in top X\% of SNPs of a continuous annotation. To partition the

128 heritability captured by various top echelons of SNPs of a given continuous annotation, we used

129 the same strategy as in a previous study ${ }^{6}$. By this strategy, the proportion of heritability

130 explained by a set of SNPs is the sum over all SNPs of the product of the $\tau^{*}$ of each category in

131 the S-LDSC model, e.g. baselineLD plus IMPACT annotation, and the SNP membership to that 
132 category ( 1 or 0 in the case of binary annotations, continuous values in the case of continuous 133 annotations) divided by the same metric for all SNPs genome-wide.

135 Conditional S-LDSC analysis to identify independent annotation-trait associations. Due to the 136 redundancy in modeled cell type programs and inherent covariance of IMPACT annotations 137 (SF2), the $\tau^{*}$ associations we find with S-LDSC cannot be independent. To this end, for each of 13895 traits across EUR and EAS for which we identified a lead IMPACT annotation, reported in 139 ST9, we performed a series of conditional analyses using S-LDSC. For each trait with more than 140 one significant $\tau^{*}$ association, we created S-LDSC models consisting of the 69 baselineLD 141 annotations, the lead annotation for that trait, and separately, each remaining significant 142 IMPACT annotation. We kept annotations that retained their $\tau^{*}$ significance when conditioned 143 on the lead annotation(s), which we also required to retain significance. We iteratively 144 performed these conditional analyses until we were no longer able to identify independent $\tau^{*}$ 145 associations.

147 Deming regression of EUR $\tau^{*}$ on EAS $\tau^{*}$. As there is significant correlation among IMPACT

148 annotations, due to redundancy in cell type regulatory elements, we used an iterative pruning 149 approach, similar to LD-pruning, to identify independent IMPACT annotations. For each trait, 150 we ranked all 707 IMPACT annotations by their $\tau^{*}$ significance values. Then, we selected the 151 lead annotation, removed all annotations correlated with Pearson $r>0.5$, and selected the next 152 lead annotation, and so on. This approach produced a set of relatively independent 153 annotations, for which the assumptions of Deming, or any, regression would not be violated. 
154 For each trait, we ran Deming regression over approximately 100 independent IMPACT

155 annotations using the $\mathrm{R}$ function deming within the package deming. Across independent

156 observations for all traits, we tested the null hypothesis that the slope of the Deming

157 regression, which considers standard errors on both the predictor (EUR $\tau^{*}$ ) and response

158 variables (EAS $\tau^{*}$ ), is equal to 1 .

160 Multi-ethnic and within-population genetic correlation. We computed the genetic correlation

$161\left(R_{g}\right)$ between pairs of 29 traits for which we acquired EUR and EAS GWAS using Popcorn

$162[\text { v.0.9.6 }]^{13}$ with default parameters, including maximum likelihood estimation as opposed to

163 regression ${ }^{14}$. First, we computed cross-population scores between the two populations using

164 the compute flag with the popcorn executable, indicating approximately the correlation

165 between LD at each SNP using EUR and EAS reference LD panels from phase 3 of 1000

166 Genomes. Then, we used the fit flag with the popcorn executable to compute the multi-ethnic

167 genetic correlation of these 29 traits. $R_{g}$ estimates computed after restricting to MAF $>5 \%$ did

168 not significantly differ from no MAF restriction. Popcorn computes $R_{g}$ using either "genetic

169 impact" (effect sizes normalized by allele frequency) or "genetic effect" (unmodified effect

170 sizes). We observed no significant heterogeneity between the $R_{g}$ computed using "genetic

171 impact" and "effect", although "genetic effect" estimates were consistently but not significantly

172 larger.

We then computed cross-trait cross-population genetic correlations across 21 traits for

174 which we observed at least one significant IMPACT annotation association in both EUR and EAS.

175 Therefore, in total we computed the genetic correlation among 42 traits ( 21 phenotypes $\times 2$ 
176 populations). For pairs of traits with one from EUR and one from EAS, we used Popcorn as

177 described above with MAF threshold of $5 \%$ and "genetic impact". For pairs of traits from the

178 same population we used LDSC [v.1.0.0]. First we used the munge_sumstats.py script to make

179 the direction of allelic effect consistent in the GWAS summary statistics while also restricting to

180 well-imputed Hapmap3 SNPs. Then, we used the Idsc.py script with the -rg flag to compute the

181 genetic correlation using EUR and EAS reference LD panels from phase 3 of 1000 Genomes

182 where appropriate.

183

184 Multi-ethnic marginal effect size correlation. We acquired GWAS summary statistics for each

185 of 21 shared traits between EUR and EAS for which there was at least one significant IMPACT

186 association in each population. Then, we restricted to SNPs shared between EUR and EAS

187 GWAS summary statistics. Next, we performed stringent iterative LD clumping with PLINK

$188[\mathrm{v} 1.90 \mathrm{~b} 3]^{15}$ using EUR summary statistics (selecting the most significant SNP, then removing all

189 SNPs in LD with $r^{2}>0.1$ within $1 \mathrm{Mb}$, then selecting the next most significant SNP, and so on).

190 This step satisfies the assumption of independence in the Pearson correlation that we will

191 compute among marginal effect sizes. We selected our initial set of SNPs under three scenarios:

192 (1) using no functional inference, (2) using the top $5 \%$ of SNPs according to the trait's lead EUR

193 IMPACT annotation, and (3) using the bottom 95\% of SNPs according to the trait's lead EUR

194 IMPACT annotation (mutually exclusive with scenario 2). With our set of independent SNPs for

195 each trait and under each of three scenarios, we compute a Pearson correlation between the

196 estimated effect sizes, while further stratifying loci on 17 EUR $P$-values $(1,0.3,0.1,0.03,0.01$, 
$3 e-3,1 e-3,3 e-4,1 e-4,3 e-5,1 e-5,3 e-6,1 e-6,3 e-7,1 e-7,3 e-8,1 e-8)$. For example, stratum with $P=0.1$ includes all SNPs with EUR GWAS $P<0.1$.

Polygenic risk score calculation. In this study, we utilized pruning and thresholding $(P+T)$ for the calculation of PRS. We constructed PRS models from either EUR summary statistics or EAS summary statistics and evaluated their predictive performance on individual EAS phenotypes. Here, we define within-population PRS as PRSEAS and trans-ethnic PRS as PRSEUR to avoid confusion. For PRSEUR, we utilized genome-wide summary statistics from EUR as reported in their publicly available version. For PRSEAS, we held out 5,000 individuals for PRS analysis and conducted GWAS using the remaining individuals to avoid overfitting (see next section). For each trait separately, we restricted our analysis to variants that exist in both GWAS summary statistics and post-imputation genotype data of EAS individuals used for PRS analysis

209 (imputation quality of $r^{2}>0.3$ in minimac3). A detailed description related to the genotyping 210 platform and imputation strategy is provided in a previous report ${ }^{2}$. We excluded the MHC 211 region in this analysis. We designed PRS models using two strategies: standard PRS and functionally-informed

213 PRS. For standard PRSEUR, we performed conventional LD clumping to acquire sets of

214 independent SNPs using EUR LD reference panels from phase3 of 1000 Genomes. Similarly for 215 PRSEAS, we utilized EAS LD reference panels from phase 3 of 1000 Genomes. We used PLINK $216[\mathrm{v} 1.90 \mathrm{~b} 3]^{15}$ to remove variants in LD with $r^{2}>0.2$ with a significance threshold for index SNPs 217 of $P=0.5$. For functionally-informed PRS, we restricted the analysis to variants with high 218 IMPACT score according to the lead IMPACT annotation before conducting LD clumping. As 
219 before, we define the lead annotation as the one with the largest $\tau^{*}$ estimate that was

220 significantly greater than 0 . When we designed PRSEUR, we utilized the lead IMPACT annotation

221 in EUR GWAS summary statistics (EAS summary statistics were not taken into account to avoid

222 overfitting). Similarly, when we design PRSEAS, we utilized the lead IMPACT annotation in EAS

223 GWAS summary statistics for which 5,000 EAS individuals for PRS analysis were removed to

224 avoid overfitting. We performed LD clumping using variants within a predefined top percentage

225 of IMPACT scores. This was determined by the percentage that captured the closest to $50 \%$ of

226 total trait heritability; considered percentages included the top $1 \%, 5 \%, 10 \%$, and $50 \%$.

227 We evaluated PRS performance using EAS individuals. First, we used all individuals in the

228 BBJ cohort for PRSEUR testing. Second, we compared the improvement afforded by IMPACT in

229 PRSEUR relative to PRSEAS models using 5,000 randomly selected individuals in BBJ; specifically

230 for case-control GWAS, we randomly selected 1,000 cases and 4,000 controls.

231 For all models, we built a PRS for each individual $j$ in our test set (in all cases, there is no

232 overlap between GWAS samples and PRS samples) using variant effect size estimates from

233 GWAS as follows:

234

$$
P R S_{j}=\sum_{i}^{M} A_{i} * \beta_{i}
$$

(Equation 1)

235

236 Where $\mathrm{M}$ is the total number of SNPs shared between GWAS summary statistics and post-

237 imputation genotype data of EAS individuals, $i$ is the $i^{\text {th }}$ SNP in the model, $A_{i}$ is the allele dosage

238 of the trait-increasing allele $i$, and $\beta_{i}$ is the estimated effect size of allele $i$ from the GWAS. We

239 calculated PRS using PLINK2. 
For QC of quantitative phenotypes, we excluded (1) related samples (PI_HAT > 0.187

241 estimated by PLINK), (2) samples with age $<18$ and age $>85$, and (3) samples with measured

242 values outside three interquartile ranges (IQR) of the upper or lower quartiles. The effect of sex,

243 age, $a g e^{2}$, the top 10 PCs, and affection status of 47 diseases were removed by linear

244 regression, and the residuals were further normalized by the rank-based inverse normal

245 transformation (see Equation 3 below). For QC of case/control phenotypes, we excluded (1)

246 related samples (PI_HAT > 0.187 estimated by PLINK) and (2) samples with age < 18 and age >

24785.

248

We then regressed our phenotype of interest $(\mathrm{Y})$, a measured quantitative trait or a

249 diagnosed disease among the PRS samples, on the per-individual PRS as follows:

250

251

252

253

254

For diseases,

$Y_{j} \sim P R S_{j}+$ sex + age + Geno $P C 1+\ldots+$ Geno $P C 10$.

(Equation 2)

For quantitative traits,

Normalized $Y_{j} \sim P R S_{j}$.

(Equation 3)

255

256

We then report the variance explained; for quantitative traits, this is the variance

257 explained by a linear model and for diseases, the variance explained is from a logistic model

258 (Nagelkerke $\left.R^{2}\right)^{14,16,17}$ which we convert to liability scale pseudo $R^{2}$ such that $R^{2}$ values are

259 comparable among both quantitative and case/control phenotypes. We used various GWAS $P$

value thresholds $(0.1,0.03,0.01,0.003,0.001,3 e-4,1 e-4,3 e-5,1 e-5)$ to assess the predictive 
261 performance of our PRS. For each model, we reported in the text the largest $R^{2}$ achieved across

262 the nine $\mathrm{P}$ value thresholds.

263 To estimate confidence intervals of PRS performance $\left(R^{2}\right.$, as explained above), we

264 conducted 1,000 bootstraps using the R package boot. We also conducted 10,000 bootstraps to 265 evaluate whether the $R^{2}$ difference between two PRS models (functionally-informed - standard)

266 is significantly greater than 0; we calculated the $R^{2}$ difference between two PRS models in each

267 round of bootstrapping (delta $R^{2}$ ), and assess its distribution in 10,000 bootstraps. If we let $\mathrm{N}$

268 be the frequency of delta $R^{2}<0$, we define one-sided $P$ values for delta $R^{2}>0$ as (N+

269 1)/10,000.

270

271 Genome-wide association studies in BBJ. As described in the previous section, we held out

2725,000 randomly selected individuals for the PRS analysis and performed GWAS on the

273 remaining individuals (sample sizes are provided in ST13-14). GWAS was conducted with PLINK2

274 using the same imputed dosages as used in the PRS analysis. For quantitative traits, normalized

275 residuals were analyzed by a linear regression model. For diseases, affection status was

276 analyzed by a logistic regression model using age, sex, and the top 10 genotype PCs as

277 covariates.

278

279 Online Methods References

280 1. Kawakami, E., Nakaoka, S., Ohta, T. \& Kitano, H. Weighted enrichment method for

281 prediction of transcription regulators from transcriptome and global chromatin

282 immunoprecipitation data. Nucleic Acids Res. 44, 5010-5021 (2016). 
283 2. Ishigaki, K., Akiyama, M., Kanai, M. \& Takahashi, A. Large scale genome-wide association 284 study in a Japanese population identified 45 novel susceptibility loci for 22 diseases. 285 bioRxiv (2019).

286 3. Amariuta, T. et al. IMPACT: Genomic Annotation of Cell-State-Specific Regulatory Elements 287 Inferred from the Epigenome of Bound Transcription Factors. Am. J. Hum. Genet. 104, $288 \quad$ 879-895 (2019).

289 4. ENCODE Project Consortium. An integrated encyclopedia of DNA elements in the human 290 genome. Nature 489, 57-74 (2012).

291 5. Kanai, M. et al. Genetic analysis of quantitative traits in the Japanese population links cell 292 types to complex human diseases. Nat. Genet. 50, 390-400 (2018).

293 6. Gazal, S. et al. Linkage disequilibrium-dependent architecture of human complex traits 294 shows action of negative selection. Nat. Genet. 49, 1421-1427 (2017).

295 7. Finucane, H. K. et al. Partitioning heritability by functional annotation using genome-wide 296 association summary statistics. Nat. Genet. 47, 1228-1235 (2015).

297 8. Finucane, H. K. et al. Heritability enrichment of specifically expressed genes identifies 298 disease-relevant tissues and cell types. Nat. Genet. 50, 621-629 (2018).

299 9. Gibbs, R. A. et al. A global reference for human genetic variation. Nature 526, 68-74 $300 \quad$ (2015).

301 10. Nagai, A. et al. Overview of the BioBank Japan Project: Study design and profile. J. $302 \quad$ Epidemiol. 27, S2-S8 (2017).

303 11. Hirata, M. et al. Cross-sectional analysis of BioBank Japan clinical data: A large cohort of 304200,000 patients with 47 common diseases. J. Epidemiol. 27, S9-S21 (2017). 
305 12. Heinz, S. et al. Simple combinations of lineage-determining transcription factors prime cis-

306 regulatory elements required for macrophage and B cell identities. Mol. Cell 38, 576-589

307 (2010).

308 13. Brown, B. C., Ye, C. J., Price, A. L., Zaitlen, N. \& Asian Genetic Epidemiology Network-Type 2

309 Diabetes. Transethnic genetic correlation estimates from summary statistics.

310 doi:10.1101/036657

311 14. Martin, A. R. et al. Clinical use of current polygenic risk scores may exacerbate health

312 disparities. Nat. Genet. 51, 584-591 (2019).

313 15. Purcell, S. et al. PLINK: a tool set for whole-genome association and population-based

$314 \quad$ linkage analyses. Am. J. Hum. Genet. 81, 559-575 (2007).

315 16. Lam, M. et al. Comparative genetic architectures of schizophrenia in East Asian and

$316 \quad$ European populations. doi:10.1101/445874

317 17. Lee, S. H., Goddard, M. E., Wray, N. R. \& Visscher, P. M. A better coefficient of

318 determination for genetic profile analysis. Genet. Epidemiol. 36, 214-224 (2012). 